\title{
VECTOR BUNDLES OVER CLASSIFYING SPACES OF $p$-LOCAL FINITE GROUPS AND BENSON-CARLSON DUALITY
}

\author{
JOSÉ CANTARERO, NATÀLIA CASTELLANA, AND LOLA MORALES
}

\begin{abstract}
In this paper we obtain a description of the Grothendieck group of complex vector bundles over the classifying space of a $p$-local finite group $(S, \mathcal{F}, \mathcal{L})$ in terms of representation rings of subgroups of $S$. We also prove a stable elements formula for generalized cohomological invariants of $p$-local finite groups, which is used to show the existence of unitary embeddings of $p$-local finite groups. Finally, we show that the augmentation $C^{*}\left(|\mathcal{L}|_{p}^{\wedge} ; \mathbb{F}_{p}\right) \rightarrow$ $\mathbb{F}_{p}$ is Gorenstein in the sense of Dwyer-Greenlees-Iyengar and obtain some consequences about the cohomology ring of $|\mathcal{L}|_{p}^{\wedge}$.
\end{abstract}

\section{INTRODUCTION}

Let $\mathbb{K}(X)$ be the Grothendieck group of the monoid of complex vector bundles over $X$. When $P$ is a finite $p$-group, Dwyer and Zabrodsky [11] showed that there is an isomorphism $\mathbb{K}(B P) \cong R(P)$, where $R(P)$ denotes the representation ring of $P$. A few years later, Jackowski and Oliver proved in [17 that if $G$ is a compact Lie group, there is an isomorphism

$$
\mathbb{K}(B G) \stackrel{\cong}{\longrightarrow} \lim R(P)
$$

where the inverse limit is taken over all $p$-toral subgroups (for all primes) with respect to inclusion and conjugation. Note that this statement has a clear 'local' flavour in the sense that this object depends only on a family of subgroups of $G$ and conjugacy relations among them.

The notions of $p$-local finite groups [6] and $p$-local compact groups [7] introduced by Broto, Levi and Oliver model the $p$-local information of finite and compact Lie groups, respectively. Hence one would expect to have an analogous description for the Grothendieck group of finite-dimensional complex vector bundles over their classifying spaces. In this paper we focus on $p$-local finite groups. Partial results about $p$-local compact groups in this direction were obtained by Cantarero and Castellana in [8].

Recall that a $p$-local finite group is a triple $(S, \mathcal{F}, \mathcal{L})$, where $S$ is a finite $p$-group, $\mathcal{F}$ is a saturated fusion system over $S$ and $\mathcal{L}$ is a centric linking system associated to $\mathcal{F}$. The classifying space of $(S, \mathcal{F}, \mathcal{L})$ is $|\mathcal{L}|_{p}^{\wedge}$. More details can be found in Section 2 of this article. Our generalization in this context is the following theorem.

Theorem. Given a $p$-local finite group $(S, \mathcal{F}, \mathcal{L})$, restriction to the subgroups of $S$ gives an isomorphism

$$
\mathbb{K}\left(|\mathcal{L}|_{p}^{\wedge}\right) \stackrel{\cong}{\longrightarrow} \lim _{\mathcal{O}\left(\mathcal{F}^{c}\right)} R(P)
$$

2010 Mathematics Subject Classification. 55R35, (primary), 20D20, 20C20 (secondary).

Key words and phrases. $p$-local, fusion system, Benson-Carlson duality. 
Hence the theorem above can be rephrased to say that $\mathbb{K}\left(|\mathcal{L}|_{p}^{\wedge}\right)$ can be computed by stable elements. An important component in the proof of this theorem is the fact that $p$-adic topological $K$-theory can also be computed by stable elements, since this cohomology theory describes the stable behaviour of complex vector bundles. In fact, we prove a stable elements formula for any generalized cohomology theory.

Theorem. Given a $p$-local finite group $(S, \mathcal{F}, \mathcal{L})$, there is an isomorphism

$$
h^{*}\left(|\mathcal{L}|_{p}^{\wedge}\right) \cong \lim _{\mathcal{O}\left(\mathcal{F}^{c}\right)} h^{*}(B P)
$$

for any generalized cohomology theory $h^{*}$.

We note that this result was already established for cohomology with coefficients in $\mathbb{F}_{p}$ by Broto-Levi-Oliver in [6]. Motivated by the biset from [6. Proposition 5.5], Ragnarsson constructed in [27] a characteristic idempotent $\omega$ in the ring of stable self maps $\left\{\Sigma^{\infty} B S, \Sigma^{\infty} B S\right\}$ associated to the saturated fusion system $\mathcal{F}$. This idempotent determines a stable summand $\mathbb{B} \mathcal{F}$ of $\Sigma^{\infty} B S$ which is homotopy equivalent to $\Sigma^{\infty}|\mathcal{L}|_{p}^{\wedge}$ and it detects $\mathcal{F}$-stable maps into spectra, in the sense that a map $f: \Sigma^{\infty} B S \rightarrow Z$ is $\mathcal{F}$-stable if and only if $f \circ \omega \simeq f$. The theorem mentioned above is a formal consequence of the existence of $\omega$ and its properties.

In 9], Castellana and Libman study the set of homotopy classes of maps between $p$-local finite groups. In particular they construct maps into the $p$-completed classifying space of a symmetric group which extend the regular representation of the Sylow subgroup. In that case, if one embeds the symmetric group as permutation matrices in a unitary group, we obtain a map which is a monomorphism in a $p$-local homotopic sense (see Section 6 for the definition of homotopy monomorphism at the prime $p$ ).

Homotopy monomorphisms $|\mathcal{L}|_{p}^{\wedge} \rightarrow B U(m)_{p}^{\wedge}$ at the prime $p$ were also studied in 8] by the first two authors of this article, where they are called unitary embeddings. Note that [8] has some overlap with this paper, but in this case the Sylow subgroup is finite and that allows us to show the existence of unitary embeddings of $p$-local finite groups. Moreover, in Section 6 we prove the following improvement.

Theorem. Given a $p$-local finite group $(S, \mathcal{F}, \mathcal{L})$, there exists a homotopy monomorphism $|\mathcal{L}|_{p}^{\wedge} \rightarrow B S U(n)_{p}^{\wedge}$ whose homotopy fibre is a $\mathbb{F}_{p}$-finite space with Poincaré duality.

The motivation for this theorem comes from duality properties of cohomology rings of finite groups. Benson-Carlson duality for cohomology rings of finite groups [3] shows that if the $\mathbb{F}_{p}$-cohomology ring of a finite group is Cohen-Macaulay, then it is Gorenstein. On the other hand, the computation by Grbic in 14 of the $\mathbb{F}_{2}$-cohomology rings of the exotic 2-local finite groups constructed in Levi-Oliver [19] shows that these rings are Gorenstein. This suggested that an extension of Benson-Carlson duality should hold for $p$-local finite groups.

Dwyer, Greenlees and Iyengar [12] viewed Benson-Carlson duality and other phenomena in the framework of ring spectra, showing that several dualities that appear in algebra and topology are particular cases of a more general situation. From this point of view, Benson-Carlson duality is a consequence of the fact that the augmentation map $C^{*}\left(B G ; \mathbb{F}_{p}\right) \rightarrow \mathbb{F}_{p}$ is Gorenstein in the sense of Definition 8.1 in $[12$.

A careful analysis of this fact shows that it is a byproduct of having an injective homomorphism $G \rightarrow S U(n)$. In this case $S U(n) / G$ satisfies Poincaré duality, but 
more importantly, only mod $p$ Poincaré duality is needed to show the Gorenstein condition. This is the motivation for the theorem mentioned above, and this is sufficient to prove a duality theorem for cohomology rings of $p$-local finite groups.

Theorem. Let $(S, \mathcal{F}, \mathcal{L})$ be a p-local finite group. Then the augmentation map $C^{*}\left(|\mathcal{L}|_{p}^{\wedge} ; \mathbb{F}_{p}\right) \rightarrow \mathbb{F}_{p}$ is Gorenstein in the sense of Dwyer-Greenlees-Iyengar [12]. Therefore if $H^{*}\left(|\mathcal{L}|_{p}^{\wedge} ; \mathbb{F}_{p}\right)$ is Cohen-Macaulay, then it is Gorenstein.

1.1. Acknowledgements. The authors are grateful to John Greenlees for suggesting that the existence of homotopy monomorphisms should have duality consequences on the cohomology ring.

1.2. Acknowledgements of financial support. The first two authors are partially supported by FEDER/MED (grants MTM2013-42293-P and MTM2016-80439P), and by SEP-CONACYT (grant 242186). The second author acknowledges financial support from the Spanish Ministry of Economy and Competitiveness through the "María de Maeztu" Programme for Units of Excellence in R\&D (MDM2014-0445).

\section{Preliminaries on $p$-LOCAl finite Groups}

In this section, we recall the notion of a $p$-local finite group introduced by Broto, Levi and Oliver in [6. One of the ingredients is the concept of saturated fusion system introduced by Puig [26. Given subgroups $P$ and $Q$ of $S$ we denote by $\operatorname{Hom}_{S}(P, Q)$ the set of group homomorphisms $P \rightarrow Q$ that are conjugations by an element of $S$ and by $\operatorname{Inj}(P, Q)$ the set of monomorphisms from $P$ to $Q$.

Definition 2.1. A fusion system $\mathcal{F}$ over a finite $p$-group $S$ is a subcategory of the category of groups whose objects are the subgroups of $S$ and such that the set of morphisms $\operatorname{Hom}_{\mathcal{F}}(P, Q)$ between two subgroups $P$ and $Q$ satisfies the following conditions:

(a) $\operatorname{Hom}_{S}(P, Q) \subseteq \operatorname{Hom}_{\mathcal{F}}(P, Q) \subseteq \operatorname{Inj}(P, Q)$ for all $P, Q \leq S$.

(b) Every morphism in $\mathcal{F}$ factors as an isomorphism in $\mathcal{F}$ followed by an inclusion.

Definition 2.2. Let $\mathcal{F}$ be a fusion system over a $p$-group $S$.

- We say that two subgroups $P, Q \leq S$ are $\mathcal{F}$-conjugate if they are isomorphic in $\mathcal{F}$.

- A subgroup $P \leq S$ is fully centralized in $\mathcal{F}$ if $\left|C_{S}(P)\right| \geq\left|C_{S}\left(P^{\prime}\right)\right|$ for all $P^{\prime} \leq S$ which are $\mathcal{F}$-conjugate to $P$.

- A subgroup $P \leq S$ is fully normalized in $\mathcal{F}$ if $\left|N_{S}(P)\right| \geq\left|N_{S}\left(P^{\prime}\right)\right|$ for all $P^{\prime} \leq S$ which are $\mathcal{F}$-conjugate to $P$.

- $\mathcal{F}$ is a saturated fusion system if the following conditions hold:

(I) Each fully normalized subgroup $P \leq S$ is fully centralized and the

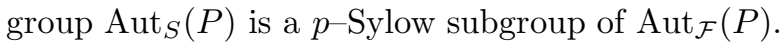

(II) If $P \leq S$ and $\varphi \in \operatorname{Hom}_{\mathcal{F}}(P, S)$ are such that $\varphi P$ is fully centralized, and if we set

$$
N_{\varphi}=\left\{g \in N_{S}(P) \mid \varphi c_{g} \varphi^{-1} \in \operatorname{Aut}_{S}(\varphi P)\right\},
$$

then there is $\bar{\varphi} \in \operatorname{Hom}_{\mathcal{F}}\left(N_{\varphi}, S\right)$ such that $\bar{\varphi}_{\mid P}=\varphi$. 
The motivating example for this definition is the fusion system of a finite group $G$. For a fixed Sylow $p$-subgroup $S$ of $G$, let $\mathcal{F}_{S}(G)$ be the fusion system over $S$ defined by setting $\operatorname{Hom}_{\mathcal{F}_{S}(G)}(P, Q)=\operatorname{Hom}_{G}(P, Q)$. This is a saturated fusion system.

In the following definition we use the notation $\operatorname{Out}_{\mathcal{F}}(P)=\operatorname{Aut}_{\mathcal{F}}(P) / \operatorname{Inn}(P)$.

Definition 2.3. Let $\mathcal{F}$ be a fusion system over a $p$-group $S$.

- A subgroup $P \leq S$ is $\mathcal{F}$-centric if $P$ and all its $\mathcal{F}$-conjugates contain their $S$-centralizers.

- A subgroup $P \leq S$ is $\mathcal{F}$-radical if $\operatorname{Out}_{\mathcal{F}}(P)$ is $p$-reduced, that is, if $\operatorname{Out}_{\mathcal{F}}(P)$ has no proper normal $p$-subgroup.

We will use $\mathcal{F}^{c}$ to denote the full subcategory of $\mathcal{F}$ whose objects are the $\mathcal{F}$ centric subgroups and $\mathcal{F}^{c r}$ for the full subcategory of $\mathcal{F}$-centric, $\mathcal{F}$-radical subgroups.

The following theorem is a version of Alperin's fusion theorem for saturated fusion systems (Theorem A.10 in [6]).

Theorem 2.4. Let $\mathcal{F}$ be a saturated fusion system over $S$. Then for each isomorphism $P \rightarrow P^{\prime}$ in $\mathcal{F}$, there exist sequences of subgroups of $S$,

$$
P=P_{0}, P_{1}, \ldots, P_{k}=P^{\prime} \quad \text { and } \quad Q_{1}, Q_{2}, \ldots, Q_{k}
$$

and morphisms $\varphi_{i} \in \operatorname{Aut}_{\mathcal{F}}\left(Q_{i}\right)$ such that the following hold.

(1) $Q_{i}$ is fully normalized, $\mathcal{F}$-centric and $\mathcal{F}$-radical for each $i$.

(2) $P_{i-1}, P_{i} \leq Q_{i}$ and $\varphi_{i}\left(P_{i-1}\right)=P_{i}$ for each $i$.

(3) $\varphi=\varphi_{k} \circ \varphi_{k-1} \circ \cdots \circ \varphi_{1}$.

The notion of a centric linking system is the extra structure needed in the definition of a $p$-local finite group to obtain a classifying space which behaves like $B G_{p}^{\wedge}$ for a finite group $G$.

Definition 2.5. Let $\mathcal{F}$ be a fusion system over a $p$-group $S$. A centric linking system associated to $\mathcal{F}$ is a category $\mathcal{L}$ whose objects are the $\mathcal{F}$-centric subgroups of $S$, together with a functor

$$
\pi: \mathcal{L} \longrightarrow \mathcal{F}^{c}
$$

and "distinguished" monomorphisms $P \stackrel{\delta_{P}}{\longrightarrow} \operatorname{Aut}_{\mathcal{L}}(P)$ for each $\mathcal{F}$-centric subgroup $P \leq S$, which satisfy the following conditions.

(A) $\pi$ is the identity on objects and surjective on morphisms. More precisely, for each pair of objects $P, Q$ in $\mathcal{L}, Z(P)$ acts freely on $\operatorname{Mor}_{\mathcal{L}}(P, Q)$ by composition (upon identifying $Z(P)$ with $\delta_{P}(Z(P)) \leq \operatorname{Aut}_{\mathcal{L}}(P)$ ) and $\pi$ induces a bijection

$$
\operatorname{Mor}_{\mathcal{L}}(P, Q) / Z(P) \stackrel{\cong}{\longrightarrow} \operatorname{Hom}_{\mathcal{F}}(P, Q)
$$

(B) For each $\mathcal{F}$-centric subgroup $P \leq S$ and each $g \in P$, the functor $\pi$ sends $\delta_{P}(g)$ to $c_{g} \in \operatorname{Aut}_{\mathcal{F}}(P)$. 
(C) For each $f \in \operatorname{Mor}_{\mathcal{L}}(P, Q)$ and each $g \in P$, the following square commutes in $\mathcal{L}$

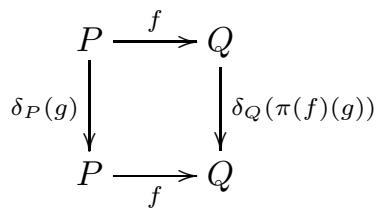

Definition 2.6. A $p$-local finite group is a triple $(S, \mathcal{F}, \mathcal{L})$, where $\mathcal{F}$ is a saturated fusion system over a finite $p$-group $S$ and $\mathcal{L}$ is a centric linking system associated to $\mathcal{F}$. The classifying space of the $p$-local finite group $(S, \mathcal{F}, \mathcal{L})$ is the space $|\mathcal{L}|_{p}^{\wedge}$.

A theorem of Chermak [10] (see also Oliver 25]) states that every saturated fusion system admits a centric linking system and that it is unique up to isomorphism of centric linking systems. In particular, the classifying space of a $p$-local finite group is determined up to homotopy equivalence by the saturated fusion system.

In many cases it is convenient to restrict the fusion system to certain subcategories. It was shown in Broto-Castellana-Grodal-Levi-Oliver [5] that one can consider certain full subcategories $\mathcal{L}_{0}$ of $\mathcal{L}$, such that the inclusion functor $\mathcal{L}_{0} \rightarrow \mathcal{L}$ induces a mod $p$ homotopy equivalence on nerves. In particular, one such is the full subcategory of $\mathcal{F}$-centric $\mathcal{F}$-radical subgroups of $S$.

Our main goal is to understand maps between classifying spaces. The key tool when studying maps between classifying spaces is the existence of $\bmod p$ homology decompositions. That is, we can reconstruct the classifying space of a $p$-local finite group up to $p$-completion as a homotopy colimit of classifying spaces of $p$-subgroups over the orbit category, $\mathcal{O}(\mathcal{F})$. The orbit category is the category whose objects are the subgroups of $S$ and whose morphisms are

$$
\operatorname{Mor}_{\mathcal{O}(\mathcal{F})}(P, Q)=\operatorname{Rep}_{\mathcal{F}}(P, Q) \stackrel{\text { def }}{=} \operatorname{Inn}(Q) \backslash \operatorname{Hom}_{\mathcal{F}}(P, Q) .
$$

Also, $\mathcal{O}\left(\mathcal{F}^{c}\right)$ is the full subcategory of $\mathcal{O}(\mathcal{F})$ whose objects are the $\mathcal{F}$-centric subgroups of $S$. The next proposition is part of Proposition 2.2 in [6].

Proposition 2.7. Fix a saturated fusion system $\mathcal{F}$ and an associated centric linking system $\mathcal{L}$, and let $\tilde{\pi}: \mathcal{L} \rightarrow \mathcal{O}\left(\mathcal{F}^{c}\right)$ be the projection functor. Let

$$
\widetilde{B}: \mathcal{O}\left(\mathcal{F}^{c}\right) \longrightarrow \text { Top }
$$

be the left Kan extension along $\widetilde{\pi}$ of the constant functor $\mathcal{L} \rightarrow$ Top that sends every object to the one-point topological space. Then $\widetilde{B}$ is a homotopy lifting of the homotopy functor $P \mapsto B P$, and

$$
|\mathcal{L}| \simeq \underset{\mathcal{O}\left(\mathcal{F}^{c}\right)}{\operatorname{hocolim}} \widetilde{B}
$$

\section{Complex Representations of Fusion systems}

Let $(S, \mathcal{F}, \mathcal{L})$ be a given $p$-local finite group. In this section we study complex representations of the finite $p$-group $S$ which are compatible with the morphisms in the fusion category $\mathcal{F}$ in a sense that will be made precise. This description coincides with the one given in Section 3 of Cantarero-Castellana [8. When dealing with a finite group $G$, this is described in Jackson [18]. 
Given a finite group $G$, we denote by $\operatorname{Rep}_{n}(G)=\operatorname{Rep}(G, U(n))$ the set of isomorphism classes of $n$-dimensional complex representations of $G$. If $\rho$ is an $n^{-}$ dimensional representation of $G$, then $\chi_{\rho}$ denotes its associated character function.

Definition 3.1. Let $\mathcal{F}$ be a fusion system over $S$. An $n$-dimensional complex representation $\rho$ of $S$ is fusion-preserving if $\rho_{\mid P}=\rho_{\mid f(P)} \circ f$ in $\operatorname{Rep}_{n}(P)$ for any $P \leq S$ and any $f \in \operatorname{Hom}_{\mathcal{F}}(P, S)$.

We denote by $\operatorname{Rep}_{n}(\mathcal{F})$ the set of isomorphism classes of $n$-dimensional complex representations of $S$ which are fusion-preserving. It is clear that if two representations of $S$ are isomorphic and one of them is fusion-preserving, so is the other one. The following lemma gives an alternative description of this set.

Lemma 3.2. Let $\mathcal{F}$ be a fusion system over $S$. The composition

$$
\lim _{\mathcal{F}} \operatorname{Rep}_{n}(P) \subseteq \prod_{P \leq S} \operatorname{Rep}_{n}(P) \rightarrow \operatorname{Rep}_{n}(S)
$$

of the inclusion and the projection map is injective and has image $\operatorname{Rep}_{n}(\mathcal{F})$.

Proof. Given $Q \leq S$, an element $\left(\rho_{P}\right)_{P}$ in the limit must satisfy $\rho_{Q}=\left(\rho_{S}\right)_{\mid Q}$ since the inclusion $Q \rightarrow S$ is a morphism in $\mathcal{F}$. Therefore injectivity is clear. By definition, a representation $\rho$ of $S$ is fusion-preserving if and only if the element $\left(\rho_{\mid P}\right)_{P}$ belongs to $\underset{\mathcal{F}}{\lim } \operatorname{Rep}_{n}(P)$, hence surjectivity follows.

Remark 3.3. Since two representations are isomorphic if and only if their characters are equal, we can conclude that $\rho$ is fusion-preserving if and only if $\chi_{\rho}(g)$ equals $\chi_{\rho}\left(g^{\prime}\right)$ whenever there is a morphism $f$ in $\mathcal{F}$ such that $f(g)=g^{\prime}$. Hence we can also think of $\operatorname{Rep}_{n}(\mathcal{F})$ as the set of $n$-dimensional characters of $S$ which are fusion-preserving in that sense.

Example 3.4. Let reg be the regular representation of $S$. It has the property that $\chi_{\text {reg }}(g)=0$ if $g \neq e$ and $\chi_{\text {reg }}(e)=|S|$. Using Remark 3.3, it is straightforward to check that reg $\in \operatorname{Rep}_{|S|}(\mathcal{F})$ for any fusion system over $S$. Any trivial representation of $S$ is also fusion-preserving.

Example 3.5. Let $\Sigma_{3}$ be the symmetric group on three letters, $S$ the subgroup generated by $(1,2,3)$, which is a 3 -Sylow subgroup of $\Sigma_{3}$, and $\mathcal{F}=\mathcal{F}_{S}\left(\Sigma_{3}\right)$. The trivial representation and the reduced regular representation of $S$ are fusion-preserving.

When constructing fusion-preserving representations, it may be convenient to restrict to the orbit category and to the family of centric or centric radical subgroups in $\mathcal{F}$ (see also Remark 3.2 in Cantarero-Castellana [8]).

Proposition 3.6. Let $\mathcal{F}$ be a saturated fusion system over $S$. Then

$$
\varliminf_{\mathcal{F} c r} \operatorname{Rep}_{n}(P)=\varliminf_{\mathcal{F}^{c}} \operatorname{Rep}_{n}(P)=\operatorname{Rep}_{n}(\mathcal{F})
$$

Moreover

$$
\lim _{\mathcal{O}\left(\mathcal{F}^{c r}\right)} \operatorname{Rep}_{n}(P) \cong \operatorname{Rep}_{n}(\mathcal{F})
$$

Proof. Since there are inclusions

$$
\lim _{\mathcal{F}} \operatorname{Rep}_{n}(P) \subseteq \lim _{\mathcal{F} c} \operatorname{Rep}_{n}(P) \subseteq \lim _{\mathcal{F} c r} \operatorname{Rep}_{n}(P),
$$


it is enough to show that $\varliminf_{\mathcal{F} c r}^{\lim } \operatorname{Rep}_{n}(P) \subseteq{\underset{\mathcal{F}}{\mathcal{F}}}_{\lim _{n}} \operatorname{Rep}_{n}(P)$.

Let $\rho \in \lim _{\mathcal{F} c r} \operatorname{Rep}_{n}(P)$. We have to show that $\rho_{\mid P}=\rho_{\mid P^{\prime}} \circ \varphi$ in $\operatorname{Rep}_{n}(P)$ for any subgroups $P, P^{\prime}$ of $S$ and any $\varphi \in \operatorname{Hom}_{\mathcal{F}}\left(P, P^{\prime}\right)$. Since this holds for inclusions, we may assume that $\varphi$ is an isomorphism. By Theorem 2.4 there exists a sequence of subgroups $P=P_{0}, P_{1}, \ldots, P_{k}=P^{\prime}$ of $S$ and a sequence of $\mathcal{F}$-centric radical subgroups $Q_{1}, Q_{2}, \ldots, Q_{k}$ of $S$, with $P_{i-1}, P_{i} \leq Q_{i}$, and a sequence of morphisms $\varphi_{i} \in \operatorname{Aut}_{\mathcal{F}}\left(Q_{i}\right)$ with $\varphi_{i}\left(P_{i-1}\right) \leq P_{i}$ which factor $\varphi$. Since $\rho_{\mid Q_{i}} \circ \varphi_{i}$ and $\rho_{\mid Q_{i}}$ are isomorphic representations and $P_{i-1}, P_{i} \leq Q_{i}$, the representations $\rho_{\mid P_{i}}$ and $\rho_{\mid P_{i-1}}$ are isomorphic to the respective restrictions of $\rho_{\mid Q_{i}}$. Therefore $\rho_{\mid P}$ is isomorphic to $\rho_{\mid P^{\prime}} \circ \varphi$ in $\operatorname{Rep}_{n}(P)$.

Moreover, the functor $\operatorname{Rep}_{n}: \mathcal{F}^{c r} \rightarrow$ Set factors through the orbit category $\mathcal{O}\left(\mathcal{F}^{c r}\right)$ since isomorphism classes of representations are fixed under inner automorphisms. Therefore the bijection just proved shows that

$$
\lim _{\mathcal{O}(\mathcal{F} c r)} \operatorname{Rep}_{n}(P) \cong \operatorname{Rep}_{n}(\mathcal{F})
$$

The rest of this section is devoted to a construction which provides fusionpreserving representations out of representations of the Sylow subgroup. In the spirit of induction, the idea is to 'induce' representations of the Sylow subgroup $S$ to fusion-preserving representations.

The key tool which will allow us to do this construction is the specific $(S, S)$ biset $\Omega$ constructed in the proof of Proposition 5.5 of Broto-Levi-Oliver [ 6 for any saturated fusion system $\mathcal{F}$ over $S$. This biset $\Omega$ satisfies that $\Omega_{\mid(P, S)}$ and $\Omega_{\mid(\varphi, S)}$ are isomorphic as $(P, S)$-bisets for any given $\varphi \in \operatorname{Hom}_{\mathcal{F}}(P, Q)$. Recall that $\Omega_{\mid(\varphi, S)}$ stands for the $(P, S)$-biset whose underlying set is $\Omega$ with the same right action of $S$ and the left action of $P$ given by

$$
p \cdot x=\varphi(p) x
$$

where the action on the right hand side is the original right action of $S$. Hence there exists an isomorphism of $(P, S)$-bisets $\tau_{\varphi}: \Omega \rightarrow \Omega$ where the action on the source is via $\varphi$, that is, $\tau_{\varphi}(\varphi(p) x)=p \tau_{\varphi}(x)$.

In the remainder of the section we use $\mathbb{C}[X]$ to denote the complex vector space with the set $X$ as basis. If $S$ acts linearly on the complex vector spaces $V$ and $W$ on the right and the left, respectively, we denote by $V \otimes_{S} W$ their tensor product as $\mathbb{C} S$-modules. Note that if $V$ has an action of $R$ on the left, $V \otimes_{S} W$ inherits a left $R$-action.

Definition 3.7. Let $\rho$ be a complex $n$-dimensional representation of the Sylow subgroup $S$ and consider the vector space $\mathbb{C}[\Omega] \otimes_{S} \mathbb{C}^{n}$, where $S$ acts on $\mathbb{C}^{n}$ via $\rho$. Define $\rho^{\mathcal{F}} \in \operatorname{Rep}_{n|\Omega / S|}(S)$ to be $\mathbb{C}[\Omega] \otimes_{S} \mathbb{C}^{n}$ with the left action of $S$ inherited from the left action of $S$ on $\Omega$.

Proposition 3.8. Let $\mathcal{F}$ be a saturated fusion system over $S$ and $\rho \in \operatorname{Rep}_{n}(S)$. Then $\rho^{\mathcal{F}} \in \operatorname{Rep}_{n|\Omega / S|}(\mathcal{F})$ and $\rho$ is a subrepresentation of $\rho^{\mathcal{F}}$.

Proof. We need to show that for any $P \leq S$ and any $\varphi \in \operatorname{Hom}_{\mathcal{F}}(P, S)$, the representations $\rho_{\mid P}^{\mathcal{F}}$ and $\rho_{\mid \varphi(P)}^{\mathcal{F}} \circ \varphi$ of $P$ are isomorphic. The map $\tau_{\varphi}: \Omega \rightarrow \Omega$ considered above induces a linear map $\mathbb{C}[\Omega] \rightarrow \mathbb{C}[\Omega]$, which we also denote by $\tau_{\varphi}$. Consider 
the map

$$
\begin{gathered}
\bar{\tau}_{\varphi}: \mathbb{C}[\Omega] \otimes_{S} \mathbb{C}^{n} \longrightarrow \mathbb{C}[\Omega] \otimes_{S} \mathbb{C}^{n} \\
x \otimes v \longmapsto \tau_{\varphi}(x) \otimes v .
\end{gathered}
$$

The map $\bar{\tau}_{\varphi}$ is well defined and linear. It is bijective because $\tau_{\varphi}$ is bijective. Given $x \in \Omega$, the equalities

$$
\bar{\tau}_{\varphi}(\varphi(p)(x \otimes v))=\bar{\tau}_{\varphi}(\varphi(p) x \otimes v)=\tau_{\varphi}(\varphi(p) x) \otimes v=p \tau_{\varphi}(x) \otimes v=p \bar{\tau}_{\varphi}(x \otimes v) .
$$

show that $\bar{\tau}_{\varphi}$ is an isomorphism from $\rho_{\mid \varphi(P)}^{\mathcal{F}} \circ \varphi$ to $\rho_{\mid P}^{\mathcal{F}}$. Hence $\rho^{\mathcal{F}}$ is fusion-preserving.

Now we prove that $\rho$ is a subrepresentation of $\rho^{\mathcal{F}}$. Let $\left\{x_{1}, \ldots, x_{r}\right\} \subset \Omega$ be a set of orbit representatives for $\Omega / S$. We can assume that $x_{1}$ is the element $[e, e] \in S \times{ }_{(S, i d)} S \subset \Omega$, where $e$ is the unit of $S$. It is clear that this element satisfies $s \cdot x_{1}=x_{1} \cdot s$ for all $s \in S$.

Given a basis $\left\{v_{1}, \ldots, v_{n}\right\}$ of $\mathbb{C}^{n}$, let $V$ be the subspace of $\mathbb{C}[\Omega] \otimes_{S} \mathbb{C}^{n}$ generated by the elements $x_{1} \otimes v_{i}$ for $i=1, \ldots, n$. Note that $V$ is $S$-invariant since

$$
s\left(x_{1} \otimes v\right)=\left(s \cdot x_{1}\right) \otimes v=\left(x_{1} \cdot s\right) \otimes v=x_{1} \otimes \rho(s)(v) .
$$

Moreover, this shows that it is $S$-isomorphic to $\mathbb{C}^{n}$ with the action of $S$ via $\rho$. Therefore $\rho$ is a subrepresentation of $\rho^{\mathcal{F}}$.

Remark 3.9. In particular, given a saturated fusion system $\mathcal{F}$ over $S$, any representation $\rho \in \operatorname{Rep}_{n}(S)$ is a subrepresentation of a fusion-preserving representation.

\section{Generalized COHOMOLOGY THEORIES OF ClASSiFying SPACES OF $p$-LOCAL FINITE GROUPS}

This section contains the proof of Theorem 4.2 which relies strongly in the work of Ragnarsson [27] on the stable homotopy theory of fusion systems. We use $\{E, F\}$ to denote the set of homotopy classes of maps between the spectra $E$ and $F$.

Given a saturated fusion system $\mathcal{F}$ over $S$, Ragnarsson [27] constructs an idempotent in the ring of stable self-maps $\left\{\Sigma^{\infty} B S, \Sigma^{\infty} B S\right\}$ associated to the saturated fusion system $\mathcal{F}$. More precisely, it is shown that there exists an idempotent $\omega$ in $\left\{\Sigma^{\infty} B S, \Sigma^{\infty} B S\right\}$ which is a $\mathbb{Z}_{p}^{\wedge}$-linear combination of stable maps of the form $\Sigma^{\infty} B \varphi \circ \operatorname{tr}_{P}$, where $\operatorname{tr}_{P}: \Sigma^{\infty} B S \rightarrow \Sigma^{\infty} B P$ is the stable transfer map and $\varphi \in \operatorname{Hom}_{\mathcal{F}}(P, S)$. This idempotent satisfies

$$
\omega \circ \Sigma^{\infty} B f \simeq \omega_{\mid \Sigma^{\infty} B P}
$$

for any $P \leq S$ and any $f \in \operatorname{Hom}_{\mathcal{F}}(P, S)$. Here $\omega_{\mid \Sigma^{\infty} B P}$ denotes the composition of $w$ with the map $j_{P}^{S}: \Sigma^{\infty} B P \rightarrow \Sigma^{\infty} B S$ induced by the inclusion of $P$ in $S$.

The homotopy type of the stable summand $\mathbb{B} \mathcal{F}$ of $\Sigma^{\infty} B S$ induced by $\omega$ coincides with the homotopy type of the classifying spectrum constructed by Broto, Levi and Oliver in [6] just after Proposition 5.5. Note that $\mathbb{B} \mathcal{F}$ is $p$-complete since it is a retract of $\Sigma^{\infty} B S$.

The spectrum $\mathbb{B} \mathcal{F}$ comes equipped with the structure map of the mapping telescope $\sigma_{\mathcal{F}}: \Sigma^{\infty} B S \rightarrow \mathbb{B} \mathcal{F}$ and a transfer map $t_{\mathcal{F}}: \mathbb{B} \mathcal{F} \rightarrow \Sigma^{\infty} B S$ such that $t_{\mathcal{F}} \circ \sigma_{\mathcal{F}} \simeq \omega$ and $\sigma_{\mathcal{F}} \circ t_{\mathcal{F}} \simeq$ id (see Section 7 of [27]).

Remark 4.1. Let $\mathcal{L}$ be a centric linking system for $\mathcal{F}$ and $\Theta: B S \rightarrow|\mathcal{L}|_{p}^{\wedge}$ the canonical inclusion induced by the structure morphism $\delta_{S}: S \rightarrow \operatorname{Aut}_{\mathcal{L}}(S)$ from Definition 2.5. Proposition 10.1 in 27] shows that there is a homotopy equivalence $h: \mathbb{B} \mathcal{F} \rightarrow \Sigma^{\infty}|\mathcal{L}|_{p}^{\wedge}$ such that $h \circ \sigma_{\mathcal{F}} \simeq \Sigma^{\infty} \Theta$. 
Theorem 4.2. Let $h^{*}$ be a generalized cohomology theory. Given a p-local finite group $(S, \mathcal{F}, \mathcal{L})$, there is an isomorphism

$$
h^{*}\left(|\mathcal{L}|_{p}^{\wedge}\right) \cong \lim _{\mathcal{O}\left(\mathcal{F}^{c}\right)} h^{*}(B P)
$$

Proof. Let $Y$ be a spectrum representing the corresponding reduced cohomology theory $\widetilde{h}^{*}$, so that we have

$$
h^{n}(\mathbb{B} \mathcal{F})=\{\mathbb{B} \mathcal{F}, Y\}_{n}=\left\{\mathbb{B} \mathcal{F}, \Sigma^{-n} Y\right\} .
$$

We will actually show that $\{\mathbb{B} \mathcal{F}, Z\} \cong \lim _{\mathcal{O}\left(\mathcal{F}^{c}\right)}\{B P, Z\}$ for any spectrum $Z$.

Let us denote by $f^{*}$ the image of a map $f$ of spectra under the functor $\{-, Z\}$. We will show that $\operatorname{Im}\left(\omega^{*}\right)$ is isomorphic to both $\lim _{\mathcal{O}\left(\mathcal{F}^{c}\right)}\left\{\Sigma^{\infty} B P, Z\right\}$ and $\{\mathbb{B} \mathcal{F}, Z\}$.

Since $t_{\mathcal{F}}^{*} \sigma_{\mathcal{F}}^{*}$ is the identity, $\sigma_{\mathcal{F}}^{*}$ is injective and so its image is isomorphic to $\{\mathbb{B} \mathcal{F}, Z\}$. On the other hand, $t_{\mathcal{F}}^{*}$ is surjective and therefore the image of $\omega^{*}$ equals the image of $\sigma_{\mathcal{F}}^{*}$. In particular, the image of $\omega^{*}$ is isomorphic to $\{\mathbb{B} \mathcal{F}, Z\}$.

Now consider the map

$$
\begin{aligned}
\varphi: \operatorname{Im}\left(\omega^{*}\right) & \rightarrow \lim _{\mathcal{O}_{\left(\mathcal{F}^{c}\right)}}\left\{\Sigma^{\infty} B P, Z\right\} \\
a & \mapsto\left(a \circ j_{P}^{S}\right)_{P}
\end{aligned}
$$

It is well defined, because given $f: P \rightarrow S$ in $\mathcal{O}\left(\mathcal{F}^{c}\right)$ and $a=\omega^{*} b \in \operatorname{Im}\left(\omega^{*}\right)$ we have

$$
\left(\omega^{*} b\right) \circ \Sigma^{\infty} f=b \circ \omega \circ \Sigma^{\infty} f \simeq b \circ \omega \circ j_{P}^{S}=\left(\omega^{*} b\right) \circ j_{P}^{S} .
$$

On the other hand, given $\left(b_{P}\right)_{P}$ in the inverse limit, the map $b_{S}$ is right $\mathcal{F}$-stable in the terminology of Definition 6.1 in 27. Hence $b_{S} \circ \omega \simeq b_{S}$ by Corollary 6.4 in [27] and therefore $\omega^{*}\left(b_{S}\right)=b_{S}$. So the projection

$$
\lim _{\mathcal{O}\left(\mathcal{F}^{c}\right)}\left\{\Sigma^{\infty} B P, Z\right\} \rightarrow\left\{\Sigma^{\infty} B S, Z\right\}
$$

factors through a map $\lim _{\mathcal{O}\left(\mathcal{F}^{c}\right)}\left\{\Sigma^{\infty} B P, Z\right\} \rightarrow \operatorname{Im}\left(\omega^{*}\right)$, which is the inverse of $\varphi$.

Remark 4.3. Note that projection to the $\left\{\Sigma^{\infty} B S, Z\right\}$-factor defines an isomorphism

$$
\lim _{\mathcal{O}\left(\mathcal{F}^{c}\right)}\left\{\Sigma^{\infty} B P, Z\right\} \rightarrow\left\{\Sigma^{\infty} B S, Z\right\}^{\mathcal{F}}
$$

where $\left\{\Sigma^{\infty} B S, Z\right\}^{\mathcal{F}}$ is the subgroup of stable elements, that is, maps $f$ such that $f \circ \Sigma^{\infty} \varphi \simeq f \circ j_{P}^{S}$ for any $\varphi: P \rightarrow S$ in $\mathcal{F}$. Hence this theorem also proved that there is an isomorphism

$$
\left\{\Sigma^{\infty}|\mathcal{L}|_{p}^{\wedge}, Z\right\} \cong\left\{\Sigma^{\infty} B S, Z\right\}^{\mathcal{F}}
$$

for any spectrum $Z$. In particular

$$
h^{*}\left(|\mathcal{L}|_{p}^{\wedge}\right) \cong h^{*}(B S)^{\mathcal{F}}
$$

for any generalized cohomology theory $h^{*}$.

The following corollary will be particularly important in the next section. Recall that $p$-adic (periodic) topological $K$-theory $K^{*}\left(-; \mathbb{Z}_{p}^{\wedge}\right)$ is the generalized cohomology theory associated to the spectrum determined by $\mathbb{Z}_{p}^{\wedge} \times B U_{p}^{\wedge}$ (see Mitchell [23] for instance). 
Corollary 4.4. Let $(S, \mathcal{F}, \mathcal{L})$ be a p-local finite group. Then

$$
K^{*}\left(|\mathcal{L}|_{p}^{\wedge} ; \mathbb{Z}_{p}^{\wedge}\right) \cong \lim _{\mathcal{O}\left(\mathcal{F}^{c}\right)} K^{*}\left(B P ; \mathbb{Z}_{p}^{\wedge}\right)
$$

In particular, $K^{*}\left(|\mathcal{L}|_{p}^{\wedge} ; \mathbb{Z}_{p}^{\wedge}\right)$ is torsion-free and concentrated in even degrees.

\section{VECTOR BUNDLES OVER CLASSIFYING SPACES OF $p$-LOCAL FINITE GROUPS}

In this section we describe the Grothendieck group of complex vector bundles over the classifying space of a $p$-local finite group in terms of the fusion-preserving characters of the Sylow subgroup $S$. Our main goal is to obtain a description for $p$-local finite groups analogous to the one in Jackowski-Oliver [17. We achieve this in Theorem 5.6 which will follow from Theorem 5.3 .

When dealing with a $p$-group $P$, Dwyer and Zabrodsky prove that there is an isomorphism $\operatorname{Vect}(B P) \cong \operatorname{Rep}(P)$, where $\operatorname{Vect}(B P)$ and $\operatorname{Rep}(P)$ are the monoids of isomorphism classes of complex vector bundles over $B P$ and of complex finite dimensional representations of $P$, respectively. Therefore we obtain an isomorphism of their Grothendieck groups $\mathbb{K}(B P) \cong R(P)$.

Theorem 5.1 ([1]). There are natural bijections

$$
\operatorname{Rep}(P, U(m)) \rightarrow[B P, B U(m)] \rightarrow\left[B P, B U(m)_{p}^{\wedge}\right]
$$

given by sending a representation $\rho$ to $B \rho$ and composing with the $p$-completion map $B U(m) \rightarrow B U(m)_{p}^{\wedge}$. Moreover, the natural map

$$
B C_{U(m)}(\rho(P)) \rightarrow \operatorname{Map}(B P, B U(m))_{B \rho}
$$

induces a homotopy equivalence

$$
B C_{U(m)}(\rho(P))_{p}^{\wedge} \rightarrow \operatorname{Map}\left(B P, B U(m)_{p}^{\wedge}\right)_{B \rho} .
$$

Recall that every $p$-local finite group $(S, \mathcal{F}, \mathcal{L})$ comes equipped with a morphism $\delta_{S}: S \rightarrow \operatorname{Aut}_{\mathcal{L}}(S)$ inducing a map $\Theta: B S \rightarrow|\mathcal{L}|_{p}^{\wedge}$. Restriction defines a map $\Theta^{*}:\left[|\mathcal{L}|_{p}^{\wedge}, B U(m)_{p}^{\wedge}\right] \rightarrow\left[B S, B U(m)_{p}^{\wedge}\right]$ which factors through the inverse limit

$$
\psi_{m}:\left[|\mathcal{L}|_{p}^{\wedge}, B U(m)_{p}^{\wedge}\right] \longrightarrow \lim _{\mathcal{O}\left(\mathcal{F}^{c}\right)}\left[B P, B U(m)_{p}^{\wedge}\right]
$$

Theorem 5.1 allows us to give an algebraic description of the inverse limit above in terms of fusion-preserving $m$-dimensional representations of $S$, since

$$
\lim _{\mathcal{O}\left(\mathcal{F}^{c}\right)}[B P, B U(m)] \cong \lim _{\mathcal{O}\left(\mathcal{F}^{c}\right)} \operatorname{Rep}_{m}(P) \cong \operatorname{Rep}_{m}(\mathcal{F})
$$

Hence we may consider $\psi_{m}$ as a map

$$
\psi_{m}:\left[|\mathcal{L}|_{p}^{\wedge}, B U(m)_{p}^{\wedge}\right] \longrightarrow \operatorname{Rep}_{m}(\mathcal{F}) .
$$

We need to see how far $\psi_{m}$ stands from being injective and surjective. A general framework of obstruction theory to address this question has been developed by Wojtkowiak [29]. There is a filtration $F_{n}|\mathcal{L}|_{p}^{\wedge}$ of $|\mathcal{L}|_{p}^{\wedge}$ induced by the skeletal filtration of the nerve of $\mathcal{O}\left(\mathcal{F}^{c}\right)$ in such a way that an element in

$$
\lim _{\mathcal{O}\left(\mathcal{F}^{c}\right)}\left[B P, B U(m)_{p}^{\wedge}\right]
$$

defines a map $F_{1}|\mathcal{L}|_{p}^{\wedge} \rightarrow B U(m)_{p}^{\wedge}$ and the obstruction theory studies how to extend it inductively to $F_{n}|\mathcal{L}|_{p}^{\wedge}$. Note that the map $\psi_{m}$ constructed above corresponds to restriction along the inclusion $F_{1}|\mathcal{L}|_{p}^{\wedge} \rightarrow|\mathcal{L}|_{p}^{\wedge}$. 
In our case, given $\rho$ in $\operatorname{Rep}_{m}(\mathcal{F})$, the obstructions for $\rho$ to be in the image or to have a unique preimage lie in higher limits of the functors

$$
\begin{aligned}
F_{i}^{\rho}: \mathcal{O}\left(\mathcal{F}^{c}\right)^{\text {op }} & \rightarrow \mathbb{Z}_{(p)}-\operatorname{Mod} \\
P & \mapsto \pi_{i}\left(\operatorname{Map}\left(\widetilde{B} P, B U(m)_{p}^{\wedge}\right)_{\widetilde{B} \rho_{\mid P}}\right)
\end{aligned}
$$

where we denote by $\widetilde{B} \rho_{\mid P}$ the composition of the map $\widetilde{B} P \rightarrow B U(m)$ induced by $\rho$ and the $p$-completion map $B U(m) \rightarrow B U(m)_{p}^{\wedge}$. More precisely, the obstruction to extend a map $f: F_{n-1}|\mathcal{L}|_{p}^{\wedge} \rightarrow B U(m)_{p}^{\wedge}$ to $F_{n}|\mathcal{L}|_{p}^{\wedge}$ without changing $f$ on $F_{n-2}|\mathcal{L}|_{p}^{\wedge}$ is a class

$$
\left[E_{n}\right] \in \lim _{\mathcal{O}\left(\mathcal{F}^{c}\right)}^{n+1} \pi_{n}\left(\operatorname{Map}\left(\widetilde{B} P, B U(m)_{p}^{\wedge}\right)_{f_{P}}\right)
$$

where $f_{P}$ is the restriction of $f$ to $\widetilde{B} P$. Similarly, the obstruction to extending a homotopy between two maps $f$ and $g$ which is already defined on $F_{n-1}|\mathcal{L}|_{p}^{\wedge}$ without changing the homotopy on $F_{n-2}|\mathcal{L}|_{p}^{\wedge}$ is a class

$$
\left[U_{n}\right] \in \underset{\mathcal{O}\left(\mathcal{F}^{c}\right)}{\lim ^{n}} \pi_{n}\left(\operatorname{Map}\left(\widetilde{B} P, B U(m)_{p}^{\wedge}\right)_{f_{P}}\right)
$$

More details can be found in Section 4 of Cantarero-Castellana 8 .

Remark 5.2. Given maps $f: X \rightarrow B U(m)_{p}^{\wedge}$ and $g: X \rightarrow B U(n)_{p}^{\wedge}$, we can define their Whitney sum $f \oplus g$ to be the composition

$$
X \stackrel{(f, g)}{\longrightarrow} B U(m)_{p}^{\wedge} \times B U(n)_{p}^{\wedge} \stackrel{s}{\longrightarrow} B U(m+n)_{p}^{\wedge}
$$

where $s: B U(m)_{p}^{\wedge} \times B U(n)_{p}^{\wedge} \rightarrow B U(m+n)_{p}^{\wedge}$ is the map induced by the group homomorphism $U(m) \times U(n) \rightarrow U(m+n)$ that sends $(A, B)$ to the matrix with $A$ and $B$ as diagonal blocks.

Theorem 5.3. Let $(S, \mathcal{F}, \mathcal{L})$ be $p$-local finite group $(S, \mathcal{F}, \mathcal{L})$ and let reg be the regular representation of $S$. The map

$$
\psi_{m}:\left[|\mathcal{L}|_{p}^{\wedge}, B U(m)_{p}^{\wedge}\right] \longrightarrow \operatorname{Rep}_{m}(\mathcal{F})
$$

has the following properties:

(1) Given $\rho \in \operatorname{Rep}_{m}(\mathcal{F})$, there exists a positive integer $M$ such that $\rho \oplus$ Mreg belongs to the image of $\psi_{m+M|S|}$.

(2) If $f_{1}, f_{2}:|\mathcal{L}|_{p}^{\wedge} \rightarrow B U(m)_{p}^{\wedge}$ are such that $\psi_{m}\left(f_{1}\right)=\psi_{m}\left(f_{2}\right)$, then there exists $h:|\mathcal{L}|_{p}^{\wedge} \rightarrow B U(n)_{p}^{\wedge}$ for some $n$ such that $f_{1} \oplus h \simeq f_{2} \oplus h$ and $\psi_{n}(h)=N$ reg for some positive integer $N$.

Proof. Let $\rho$ be a fusion-preserving representation of $S$, that is, $\rho \in \operatorname{Rep}_{m}(\mathcal{F})$ for some $m>0$. For each $P \leq S$, let $\left\{\mu_{1}, \ldots, \mu_{r}\right\}$ be the set of all irreducible representations of $P$ and consider the decompositions

$$
\begin{gathered}
\rho_{\mid P}=n_{1} \mu_{1} \oplus \cdots \oplus n_{r} \mu_{r} \\
\operatorname{reg}_{\mid P}=k_{1} \mu_{1} \oplus \cdots \oplus k_{r} \mu_{r}
\end{gathered}
$$

as sums of irreducible representations of $P$. Now

$$
\operatorname{Map}\left(\widetilde{B} P, B U(m)_{p}^{\wedge}\right)_{\widetilde{B} \rho_{\mid P}} \simeq B C_{U(m)}(\rho(P))_{p}^{\wedge} \simeq \prod_{i=1}^{r} B U\left(n_{i}\right)_{p}^{\wedge}
$$


where the first equivalence follows from Dwyer-Zabrodsky [11] and the second equivalence is a consequence of Schur's Lemma. In particular, the $\operatorname{space} \operatorname{Map}\left(\widetilde{B} P, B U(m)_{p}^{\wedge}\right)_{\widetilde{B} \rho_{\mid P}}$ is simply connected.

The proof of Proposition 2.4 in [17] shows that the component of the constant map of $\operatorname{Map}\left(\widetilde{B} P, B U_{p}^{\wedge}\right)$ satisfies

$$
\pi_{i}\left(\operatorname{Map}\left(\widetilde{B} P, B U_{p}^{\wedge}\right)_{0}\right) \cong K^{-i}(\widetilde{B} P) \otimes \mathbb{Z}_{p}^{\wedge} \cong K_{P}^{-i}(\mathrm{pt}) \otimes \mathbb{Z}_{p}^{\wedge}
$$

for $i>0$ and $R(P) \otimes \mathbb{Z}_{p}^{\wedge} \cong K\left(\widetilde{B} P ; \mathbb{Z}_{p}^{\wedge}\right)$ by Lemma 2.1 in [1]. Hence, if $z: B U(m)_{p}^{\wedge} \rightarrow$ $B U_{p}^{\wedge}$ is the map induced by the inclusion $U(m) \subseteq U$, we also have for $i>0$

$$
\pi_{i}\left(\operatorname{Map}\left(\widetilde{B} P, B U_{p}^{\wedge}\right)_{z \circ \widetilde{B} \rho_{\mid P}}\right) \cong K^{-i}\left(\widetilde{B} P ; \mathbb{Z}_{p}^{\wedge}\right)
$$

because all the components of $\operatorname{Map}\left(\widetilde{B} P, B U_{p}^{\wedge}\right)$ are homotopy equivalent. Thus the mapping space $\operatorname{Map}\left(\widetilde{B} P, B U_{p}^{\wedge}\right)_{z \circ \widetilde{B} \rho_{\mid P}}$ is also simply connected. Therefore the first obstructions $\left[E_{1}\right]$ to the respective extension problems vanish. Hence there are maps from $F_{2}|\mathcal{L}|_{p}^{\wedge}$ to $B U(m)_{p}^{\wedge}$ and $B U_{p}^{\wedge}$ that extend the maps $\widetilde{B} \rho_{\mid P}$ and $z \circ \widetilde{B} \rho_{\mid P}$, respectively. These results continue to hold if we replace $\rho$ by $\rho \oplus M \operatorname{reg}$ for any $M$.

By Corollary 3.4 in Broto-Levi-Oliver [6], there exists a positive integer $N_{\mathcal{F}}$ such that the higher limits of any functor $G: \mathcal{O}\left(\mathcal{F}^{c}\right) \rightarrow \mathbb{Z}_{(p)}-$ Mod vanish above dimension $N_{\mathcal{F}}$. We can assume $N_{\mathcal{F}} \geq 2$. Since the homotopy groups of the classifying spaces of complex unitary groups stabilize to the homotopy groups of $B U$, we can find a positive integer $M>0$ such that the maps $B U\left(n_{j}+M k_{j}\right) \rightarrow B U$ induce an isomorphism on the $i$ th homotopy group for all $i \leq N_{\mathcal{F}}$ and for all $j=1, \ldots, r$. If $i$ is even, postcomposition with the map $z: B U(m+M|S|)_{p}^{\wedge} \rightarrow B U_{p}^{\wedge}$ induces a commutative diagram

$$
\begin{aligned}
& \begin{array}{c}
\pi_{i}\left(\prod_{j=1}^{r} B U\left(n_{j}+M k_{j}\right)_{p}^{\wedge}\right) \longrightarrow R(P) \otimes \mathbb{Z}_{p}^{\wedge} \\
\downarrow \cong \quad \cong \mid
\end{array} \\
& \pi_{i}\left(\operatorname{Map}\left(\widetilde{B} P, B U(m+M|S|)_{p}^{\wedge}\right)_{\widetilde{B}(\rho \oplus M \operatorname{reg})_{\mid P}}\right) \longrightarrow \pi_{i}\left(\operatorname{Map}\left(\widetilde{B} P, B U_{p}^{\wedge}\right)_{z \circ \widetilde{B}(\rho \oplus M \operatorname{reg})_{\mid P}}\right)
\end{aligned}
$$

where the top row corresponds to the map $\prod B U\left(n_{j}+M k_{j}\right)_{p}^{\wedge} \rightarrow \prod B U_{p}^{\wedge}$ induced by the inclusions $U\left(n_{j}+M k_{j}\right) \rightarrow U$ and therefore it induces an isomorphism on the $i$ th homotopy group for even $i \leq N_{\mathcal{F}}$ (see [24, Proposition A.2]). A similar argument shows that the bottom isomorphism also holds for odd $i \leq N_{\mathcal{F}}$ since these homotopy groups are all zero. Since the obstruction theory of Wojtkowiak [29] is natural with respect to postcomposition, the obstructions of one extension problem are mapped to the other.

Just as before, we have

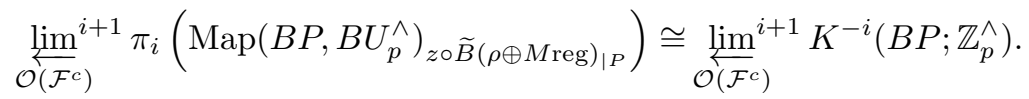

This isomorphism sends the obstructions of the extension problem with $B U_{p}^{\wedge}$ to the obstruction classes associated to the problem of existence of an element of $K^{-i}\left(|\mathcal{L}|_{p}^{\wedge} ; \mathbb{Z}_{p}^{\wedge}\right)$ that maps to the element of $\lim _{\leftarrow\left(\mathcal{F}^{c}\right)} K^{-i}\left(B P ; \mathbb{Z}_{p}^{\wedge}\right)$ determined by the 
representation $\rho \oplus M \mathrm{reg}$. Corollary 4.4 tells us that such an element exists. Therefore it is possible to construct a map $F_{N_{\mathcal{F}}+1}|\mathcal{L}|_{p}^{\wedge} \rightarrow B U_{p}^{\wedge}$ that extends the maps $z \circ \widetilde{B}(\rho \oplus M \mathrm{reg})_{\mid P}$. The obstructions of the extension problem with $B U(m+M|S|)_{p}^{\wedge}$ are mapped to the obstructions of the extension problem with $B U_{p}^{\wedge}$ via an isomorphism, so these obstructions must vanish. Hence it is possible to construct a map

$$
F_{N_{\mathcal{F}}+1}|\mathcal{L}|_{p}^{\wedge} \rightarrow B U(m+M|S|)_{p}^{\wedge}
$$

that extends the maps $\widetilde{B}(\rho \oplus M \mathrm{reg})_{\mid P}$. Finally the $\lim ^{i+1}$-term of $F_{i}^{\rho \oplus M \mathrm{reg}}$ vanishes when $i \geq N_{\mathcal{F}}$, so we can further extend it to a map $f:|\mathcal{L}|_{p}^{\wedge} \rightarrow B U(m+M|S|)_{p}^{\wedge}$ which satisfies $\psi_{m+M|S|}(f)=\rho \oplus M$ reg.

Let $f_{1}, f_{2}:|\mathcal{L}|_{p}^{\wedge} \rightarrow B U(m)_{p}^{\wedge}$ be such that $\psi_{m}\left(f_{1}\right)=\psi_{m}\left(f_{2}\right)=\rho$. By the first part applied to the regular representation of $S$, there is some $M>0$ such that the obstructions to existence vanish in each step, hence

$$
M \text { reg }=\psi_{M|S|}(h)
$$

for a certain map $h:|\mathcal{L}|_{p}^{\wedge} \rightarrow B U(M|S|)_{p}^{\wedge}$. Then we have

$$
\psi_{m+M|S|}\left(f_{1} \oplus h\right)=\rho \oplus M \mathrm{reg}=\psi_{m+M|S|}\left(f_{2} \oplus h\right) .
$$

Now we follow the same process as in the first part to construct a homotopy between $f_{1} \oplus h$ and $f_{2} \oplus h$. In this process, the first obstruction to uniqueness $\left[U_{1}\right]$ vanishes for the same reason, the obstructions up to filtration level $N_{\mathcal{F}}$ vanish because Corollary 4.4 tells us that there is a unique element in $K^{-i}\left(|\mathcal{L}|_{p}^{\wedge} ; \mathbb{Z}_{p}^{\wedge}\right)$ that maps to the element of $\lim _{\mathcal{O}\left(\mathcal{F}^{c}\right)} K^{-i}\left(B P ; \mathbb{Z}_{p}^{\wedge}\right)$ determined by the representation $\rho \oplus M$ reg. And the rest of obstructions vanish by Corollary 3.4 in Broto-Levi-Oliver $[6$.

Remark 5.4. Note that the previous theorem shows that for any $p$-local finite group $(S, \mathcal{F}, \mathcal{L})$, there exists a map $f:|\mathcal{L}|_{p}^{\wedge} \rightarrow B U(n)_{p}^{\wedge}$ such that $f_{\mid \widetilde{B} S} \simeq \widetilde{B}(M \mathrm{reg})$ for some $M>0$.

The maps $\psi_{n}$ from Theorem 5.3 assemble to define a map of monoids

$$
\coprod_{n \geq 0}\left[|\mathcal{L}|_{p}^{\wedge}, B U(n)_{p}^{\wedge}\right] \rightarrow \coprod_{n \geq 0} \operatorname{Rep}_{n}(\mathcal{F})
$$

where the monoid structure on the first set is described in Remark 5.2 and on the right hand side is given by direct sum of representations. Therefore it induces a group homomorphism between their Grothendieck groups

$$
\Psi: \mathbb{K}^{\prime}\left(|\mathcal{L}|_{p}^{\wedge}\right) \rightarrow R(\mathcal{F})
$$

such that the following diagram commutes

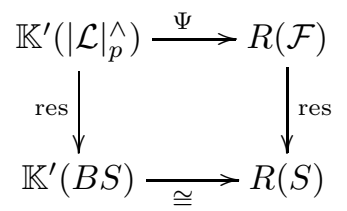

The following lemma relates this group to the Grothendieck group of complex vector bundles over $|\mathcal{L}|_{p}^{\wedge}$.

Lemma 5.5. The Grothendieck group of complex vector bundles over $|\mathcal{L}|_{p}^{\wedge}$ is isomorphic to $\mathbb{K}^{\prime}\left(|\mathcal{L}|_{p}^{\wedge}\right)$. 
Proof. Note that $p$-completion defines a map

$$
\left[|\mathcal{L}|_{p}^{\wedge}, B U(n)\right] \rightarrow\left[|\mathcal{L}|_{p}^{\wedge}, B U(n)_{p}^{\wedge}\right] .
$$

By Theorem 4.2, we have $\widetilde{H}^{k}\left(|\mathcal{L}|_{p}^{\wedge} ; \mathbb{Z}[1 / p]\right)=0$. The space $B U(n)$ is simply connected, in particular nilpotent. Moreover, pointed homotopy classes of maps into $B U(n)$ and $B U(n)_{p}^{\wedge}$ coincide with unpointed homotopy classes. Therefore Theorem 1.5 in Miller [22] shows that this map is a bijection. This defines an isomorphism of monoids

$$
\coprod_{n \geq 0}\left[|\mathcal{L}|_{p}^{\wedge}, B U(n)\right] \rightarrow \coprod_{n \geq 0}\left[|\mathcal{L}|_{p}^{\wedge}, B U(n)_{p}^{\wedge}\right]
$$

hence their Grothendieck groups are isomorphic.

Recall that $\mathbb{K}(X)$ denotes the Grothendieck group of complex vector bundles over $X$. Given the result of the previous lemma, we will abuse the notation and use $\mathbb{K}\left(|\mathcal{L}|_{p}^{\wedge}\right)$ for both Grothendieck groups. We will now show that $\Psi$ is an isomorphism.

Theorem 5.6. The map $\Psi: \mathbb{K}\left(|\mathcal{L}|_{p}^{\wedge}\right) \rightarrow R(\mathcal{F})$ is an isomorphism.

Proof. First we check that $\Psi$ is a monomorphism. Assume that we have two maps $f:|\mathcal{L}|_{p}^{\wedge} \rightarrow B U(n)_{p}^{\wedge}$ and $g:|\mathcal{L}|_{p}^{\wedge} \rightarrow B U(m)_{p}^{\wedge}$ such that $\Psi(f-g)=0$. Then we must have $f_{\mid B S}-g_{\mid B S}=0$ in $\mathbb{K}(B S)$ and so there exists $f^{\prime}: B S \rightarrow B U(k)_{p}^{\wedge}$ such that $f_{\mid B S} \oplus f^{\prime} \simeq g_{\mid B S} \oplus f^{\prime}$. This implies $m=n$. Since $\left[B S, B U(k)_{p}^{\wedge}\right] \cong \operatorname{Rep}(S, U(k))$, we can assume that $f^{\prime}$ is induced by a representation $\rho$ of $S$. By Proposition 3.8. we can assume that $\rho$ is fusion-preserving, and by Theorem 5.3 that it belongs to the image of $\psi_{k}$. Hence we can take $f^{\prime}$ to be the restriction of a map $t:|\mathcal{L}|_{p}^{\wedge} \rightarrow$ $B U(k)_{p}^{\wedge}$ and we obtain $\psi_{m+k}(f \oplus t)=\psi_{m+k}(g \oplus t)$. By Theorem 5.3, there exists $h:|\mathcal{L}|_{p}^{\wedge} \rightarrow B U(r)_{p}^{\wedge}$ for some $r>0$ such that $f \oplus t \oplus h \simeq g \oplus t \oplus h$. Therefore $f-g=0$ in $\mathbb{K}\left(|\mathcal{L}|_{p}^{\wedge}\right)$.

Let $\chi \in R(\mathcal{F})$, say $\chi=\rho_{1}-\rho_{2}$, where both $\rho_{1}$ and $\rho_{2}$ are fusion-preserving. By Theorem 5.3 there exist positive integers $k_{1}, k_{2}$ and maps $f:|\mathcal{L}|_{p}^{\wedge} \rightarrow B U\left(n_{1}\right)_{p}^{\wedge}$ and $g:|\mathcal{L}|_{p}^{\wedge} \rightarrow B U\left(n_{2}\right)_{p}^{\wedge}$ such that

$$
\psi_{n_{1}}(f)=\rho_{1}+k_{1} \text { reg } \quad \text { and } \quad \psi_{n_{2}}(g)=\rho_{2}+k_{2} \text { reg. }
$$

We can take $k_{2}$ big enough so that $\left(k_{2}-k_{1}\right)$ reg $\in \operatorname{Im}\left(\psi_{\left(k_{2}-k_{1}\right)|S|}\right)$. Then

$$
\chi=\left(\rho_{1}+k_{1} \mathrm{reg}\right)-\left(\rho_{2}+k_{2} \mathrm{reg}\right)+\left(k_{2}-k_{1}\right) \operatorname{reg} \in \operatorname{Im}(\Psi) .
$$

One could wonder at this point whether the Grothendieck construction of the monoid of fusion-preserving representations coincides with the inverse limit of representation rings over the orbit category. The answer is given by the following proposition.

Proposition 5.7. There is an isomorphism $R(\mathcal{F}) \cong \lim _{\mathcal{O}\left(\mathcal{F}^{c}\right)} R(P)$.

Proof. Consider the map

$$
\begin{aligned}
\varphi: R(\mathcal{F}) & \rightarrow \lim _{\mathcal{O}\left(\mathcal{F}^{c}\right)} R(P) \\
\chi & \mapsto\left(\operatorname{res}_{P} \chi\right)_{P}
\end{aligned}
$$


where $\operatorname{res}_{P}$ is the composition $R(\mathcal{F}) \rightarrow R(S) \rightarrow R(P)$ of the respective restriction maps. If $\chi=\rho_{1}-\rho_{2}$, where $\rho_{1}$ and $\rho_{2}$ are fusion-preserving representations of $S$, then given $f: P \rightarrow S$ in $\mathcal{O}\left(\mathcal{F}^{c}\right)$

$$
f^{*} \operatorname{res}_{S}\left(\rho_{1}-\rho_{2}\right)=f^{*}\left(\rho_{1}\right)-f^{*}\left(\rho_{2}\right)=\operatorname{res}_{P}\left(\rho_{1}\right)-\operatorname{res}_{P}\left(\rho_{2}\right)=\operatorname{res}_{P}\left(\rho_{1}-\rho_{2}\right)
$$

and so $\varphi$ is well defined. This map is clearly injective. On the other hand, given an element $\left(\chi_{P}\right)_{P}$ of the inverse limit, consider $\chi_{S}=\alpha_{1}-\alpha_{2}$. To show surjectivity, it suffices to show that $\chi_{S}$ can be written as the formal difference of two fusionpreserving representations. By Proposition 3.8 and Maschke's Lemma, there is a representation $\beta$ such that $\alpha_{2} \oplus \beta=\alpha_{2}^{\mathcal{F}}$ and $\alpha_{2}^{\mathcal{F}}$ is fusion-preserving. Then

$$
\chi_{S}=\left(\alpha_{1} \oplus \beta\right)-\alpha_{2}^{\mathcal{F}}
$$

and therefore, given $f: P \rightarrow S$ in $\mathcal{O}\left(\mathcal{F}^{c}\right)$ we have

$$
\operatorname{res}_{P}\left(\alpha_{1} \oplus \beta\right)-\operatorname{res}_{P}\left(\alpha_{2}^{\mathcal{F}}\right)=f^{*}\left(\alpha_{1} \oplus \beta\right)-f^{*}\left(\alpha_{2}^{\mathcal{F}}\right)=f^{*}\left(\alpha_{1} \oplus \beta\right)-\operatorname{res}_{P}\left(\alpha_{2}^{\mathcal{F}}\right) .
$$

That is, $\alpha_{1} \oplus \beta$ is fusion-preserving as we wanted to show.

Remark 5.8. Equivalently, the projection to the $R(S)$-component shows that we can also see $R(\mathcal{F})$ as the subring of stable elements of $R(S)$.

\section{Duality}

This section contains the proof of Theorem 6.7 that is, $C^{*}\left(|\mathcal{L}| ; \mathbb{F}_{p}\right) \rightarrow \mathbb{F}_{p}$ is Gorenstein for any $p$-local finite group $(S, \mathcal{F}, \mathcal{L})$. The motivation for this comes from extending Benson-Carlson duality 3 to cohomology rings of $p$-local finite groups. This phenomenon was already observed on the computation 14 by Grbić of the $\mathbb{F}_{2}$-cohomology rings of the exotic 2-local finite groups constructed by Levi and Oliver in [19. This suggested that an extension of Benson-Carlson duality should hold for $p$-local finite groups.

The strategy is to follow Example 10.3 of Dwyer-Greenlees-Iyengar [12, where it is shown that $C^{*}\left(B G ; \mathbb{F}_{p}\right) \rightarrow \mathbb{F}_{p}$ is Gorenstein for any finite group $G$. The main ingredient in their proof is the existence of a complex faithful representation of $G$ into some $S U(n)$ such that $H^{*}\left(S U(n) / G ; \mathbb{F}_{p}\right)$ is a Poincaré duality algebra. Our strategy is to mimic their proof using the existence of a homotopy monomorphism $|\mathcal{L}|_{p}^{\wedge} \rightarrow B S U(m)_{p}^{\wedge}$ at the prime $p$ from Theorem 6.2. The first step in our proof of Theorem 6.7 is to show that the mod $p$ cohomology of the homotopy fibre of such a map is a Poincaré duality algebra. This application of Theorem 6.2 was suggested to us by John Greenlees.

In order to prove Theorem 6.2 we recall the notion of homotopy monomorphism at the prime $p$ from Cantarero-Castellana [8].

Definition 6.1. A connected pointed space $X$ is $B \mathbb{Z} / p$-null if the pointed mapping space $\operatorname{Map}_{*}(B \mathbb{Z} / p, X)$ is contractible for any choice of basepoint in $X$. A map $f: X \rightarrow Y$ is called a homotopy monomorphism at $p$ if the homotopy fibre of $f_{p}^{\wedge}$ is $B \mathbb{Z} / p-$ null.

When the prime $p$ in question is clear, we will just write homotopy monomorphism. Recall that a space is called $\mathbb{F}_{p}$-finite if its $\mathbb{F}_{p}$-cohomology ring is a finite $\mathbb{F}_{p}$-vector space.

Theorem 6.2. There exists a homotopy monomorphism $|\mathcal{L}|_{p}^{\wedge} \rightarrow B S U(m)_{p}^{\wedge}$ for some $m>0$. 
Proof. By Theorem 5.3, there is a multiple of the regular representation of $S$ in the image of some $\psi_{n}$. A preimage of this representation must be a homotopy monomorphism $|\mathcal{L}|_{p}^{\wedge} \rightarrow B U(n)_{p}^{\wedge}$ by Theorem 2.5 from Cantarero-Castellana [8]. Consider the map $B U(n)_{p}^{\wedge} \rightarrow B S U(n+1)_{p}^{\wedge}$ induced by the standard inclusion of $U(n)$ in $S U(n+1)$. The homotopy fibre of this map is $(S U(n+1) / U(n))_{p}^{\wedge}$, which is $\mathbb{F}_{p}$-finite, so it is a homotopy monomorphism by Proposition 2.2 from [8]. Moreover, since $(S U(n+1) / U(n))_{p}^{\wedge}$ is connected, Lemma 2.4 (c) from [8] implies that the composition $|\mathcal{L}|_{p}^{\wedge} \rightarrow B S U(n+1)_{p}^{\wedge}$ of these two maps is a homotopy monomorphism.

The restriction of a homotopy monomorphism $|\mathcal{L}|_{p}^{\wedge} \rightarrow B S U(n)_{p}^{\wedge}$ to $B S$ determines a faithful fusion-preserving representation $\rho: S \rightarrow S U(n)$. Since $\rho$ is injective, we abuse the notation and use $S U(n) / P$ to denote $S U(n) / \rho(P)$ for any $P \leq S$.

For what follows, we will consider again the $(S, S)$-biset $\Omega$ from Proposition 5.5 of Broto-Levi-Oliver [6. Recall that $\Omega$ is a disjoint union of bisets of the form $S \times{ }_{(P, \varphi)} S$ with $P \leq S$ and $\varphi: P \rightarrow S$ in $\mathcal{F}$. Moreover, this set is $\mathcal{F}$-invariant in the sense that for each $P \leq S$ and each $\varphi \in \operatorname{Hom}_{\mathcal{F}}(P, S),\left.\Omega\right|_{(P, S)}$ and $\left.\Omega\right|_{(\varphi, S)}$ are isomorphic $(P, S)$-bisets.

Each $(S, S)$-biset of the form $S \times{ }_{(P, \varphi)} S$ with $\varphi \in \operatorname{Hom}_{\mathcal{F}}(P, S)$ induces an endomorphism of $H^{*}\left(S U(n) / S ; \mathbb{F}_{p}\right)$ in the following way. The representation $\rho$ is fusion-preserving, hence there exists $A \in S U(n)$ such that $A \rho(p) A^{-1}=\rho(\varphi(p))$ for all $p \in P$. We define

$$
\begin{aligned}
\bar{\varphi}: S U(n) / P & \rightarrow S U(n) / S \\
x P & \mapsto A x A^{-1} S
\end{aligned}
$$

This map is well defined and it does not depend on the choice of $A$ up to homotopy. To see this, note that any two choices differ by an element in $C_{S U(n)}(\rho(P))$, which can be regarded as the fibre of the determinant $C_{U(n)}(\rho(P)) \rightarrow S^{1}$ over 1 . The centralizer in $U(n)$ is a product of unitary groups by Schur's lemma, hence pathconnected. The determinant $C_{U(n)}(\rho(P)) \rightarrow S^{1}$ induces an epimorphism on the fundamental group since this centralizer is a product of unitary groups and the determinant $U(m) \rightarrow S^{1}$ induces an isomorphism on the fundamental group. By the long exact sequence of homotopy groups, we conclude that $C_{S U(n)}(\rho(P))$ is pathconnected. Therefore any two choices are connected by a path, which determines a homotopy between the two maps they would define.

Let $\operatorname{tr}_{P}^{S}$ be the transfer in cohomology with coefficients in $\mathbb{F}_{p}$ associated to the covering map $i_{P}: S U(n) / P \rightarrow S U(n) / S$. Then we have an endomorphism of $H^{*}\left(S U(n) / S ; \mathbb{F}_{p}\right)$ defined by

$$
\bar{\Omega}^{*}=\sum_{(P, \varphi) \in \Omega} n_{P, \varphi} \operatorname{tr}_{P}^{S} \bar{\varphi}^{*}
$$

where $(P, \varphi) \in \Omega$ means $\Omega$ has a direct summand of the form $S \times_{(P, \varphi)} S$ and $n_{P, \varphi}$ is the number of times this summand appears.

The proof of Proposition 6.5 requires the following technical lemma.

Lemma 6.3. The image of the endomorphism $\bar{\Omega}^{*}$ is isomorphic to the inverse limit over $\mathcal{O}\left(\mathcal{F}^{c}\right)$ of the cohomology groups $H^{*}\left(S U(n) / P ; \mathbb{F}_{p}\right)$. 
Proof. Consider the map

$$
\begin{aligned}
g: \operatorname{Im}\left(\bar{\Omega}^{*}\right) & \rightarrow \lim _{\mathcal{O}\left(\mathcal{F}^{c}\right)} H^{*}\left(S U(n) / P ; \mathbb{F}_{p}\right) \\
x & \mapsto\left(i_{P}^{*} x\right)_{P}
\end{aligned}
$$

where $i_{P}: S U(n) / P \rightarrow S U(n) / S$ is the covering map introduced above. Given $\varphi: P \rightarrow Q$ in $\mathcal{O}\left(\mathcal{F}^{c}\right)$ we have

$$
\varphi^{*} i_{Q}^{*} \bar{\Omega}^{*}=i_{P}^{*} \bar{\Omega}^{*}
$$

because $\Omega$ is $\mathcal{F}$-invariant. Hence $g$ is well defined. Given an element $\left(x_{P}\right)_{P}$ in the inverse limit, we have

$$
\bar{\Omega}^{*}\left(x_{S}\right)=\sum_{(P, \varphi) \in \Omega} n_{P, \varphi} \operatorname{tr}_{P}^{S} \bar{\varphi}^{*}\left(x_{S}\right)=\sum_{(P, \varphi) \in \Omega} n_{P, \varphi} \operatorname{tr}_{P}^{S} i_{P}^{*}\left(x_{S}\right)
$$

because $\left(x_{P}\right)_{P}$ belongs to the limit and

$$
\sum_{(P, \varphi) \in \Omega} n_{P, \varphi} \operatorname{tr}_{P}^{S} i_{P}^{*}\left(x_{S}\right)=\sum_{(P, \varphi) \in \Omega} n_{P, \varphi}[S: P] x_{S}=\frac{|\Omega|}{|S|} x_{S}=x_{S}
$$

because the biset $\Omega$ satisfies $|\Omega| /|S| \equiv 1(\bmod p)$ (see Proposition 5.5 (c) in [6]). So $x_{S}$ belongs to the image of $\bar{\Omega}^{*}$ and $g\left(x_{S}\right)=\left(x_{P}\right)_{P}$. This shows that $g$ is surjective. Injectivity is clear.

Remark 6.4. Note that projection to $H^{*}\left(S U(n) / S ; \mathbb{F}_{p}\right)$ gives an isomorphism

$$
\lim _{\mathcal{O}\left(\mathcal{F}^{c}\right)} H^{*}\left(S U(n) / P ; \mathbb{F}_{p}\right) \rightarrow H^{*}\left(S U(n) / S ; \mathbb{F}_{p}\right)^{\mathcal{F}}
$$

to the direct summand of $\mathcal{F}$-stable elements in $H^{*}\left(S U(n) / S ; \mathbb{F}_{p}\right)$, that is, elements $x$ such that $\bar{\varphi}^{*} x=i_{P}^{*} x$ for any $\varphi: P \rightarrow S$ in $\mathcal{F}$. The proof of Lemma 6.3 shows that $\bar{\Omega}^{*}$ is the identity on this summand. Moreover, the endomorphism $\bar{\Omega}^{*}$ is $H^{*}\left(S U(n) / S ; \mathbb{F}_{p}\right)^{\mathcal{F}}$-linear. Given elements $r$ in $H^{*}\left(S U(n) / S ; \mathbb{F}_{p}\right)^{\mathcal{F}}$ and $x$ in $H^{*}\left(S U(n) / S ; \mathbb{F}_{p}\right)$, then

$$
\operatorname{tr}_{P}^{S} \bar{\varphi}^{*}(r x)=\operatorname{tr}_{P}^{S}\left(i_{P}^{*}(r) \cdot \bar{\varphi}^{*}(x)\right)=r \cdot \operatorname{tr}_{P}^{S}\left(\bar{\varphi}^{*}(x)\right),
$$

where the first equality is due to the fact that $r$ is stable and the second equality holds by Frobenius reciprocity.

Proposition 6.5. Let $F$ be the homotopy fibre of a homotopy monomorphism from $|\mathcal{L}|_{p}^{\wedge}$ to $B S U(n)_{p}^{\wedge}$ at the prime $p$. Then there is an additive isomorphism

$$
H^{*}\left(F ; \mathbb{F}_{p}\right) \cong \lim _{\mathcal{O}\left(\mathcal{F}^{c}\right)} H^{*}\left(S U(n) / P ; \mathbb{F}_{p}\right) .
$$

Moreover, $S U(n) / S$ is an orientable manifold and the cohomological fundamental class $\omega_{S} \in H^{*}\left(S U(n) / S ; \mathbb{F}_{p}\right)$ is a stable element.

Proof. For each $P \leq S$, consider the cohomological Serre spectral sequence $E_{*}(P)$ associated to the fibration $S U(n) \rightarrow S U(n) / P \rightarrow B P$. More precisely, this spectral sequence comes from the double complex

$$
C^{r, s}(P)=\operatorname{Hom}_{\mathbb{F}_{p}[P]}\left(M_{r}, C^{s}\left(S U(n) ; \mathbb{F}_{p}\right)\right)
$$


where $M_{*}$ is a free resolution of $\mathbb{F}_{p}$ as a $\mathbb{F}_{p}[S]$-module and $P$ acts on the singular cochains $C^{*}\left(S U(n) ; \mathbb{F}_{p}\right)$ via the linear representation $\rho$. There is a transfer

$$
\operatorname{tr}_{P}^{S}: \operatorname{Hom}_{\mathbb{F}_{p}[P]}\left(M_{r}, C^{s}\left(S U(n) ; \mathbb{F}_{p}\right)\right) \rightarrow \operatorname{Hom}_{\mathbb{F}_{p}[S]}\left(M_{r}, C^{s}\left(S U(n) ; \mathbb{F}_{p}\right)\right)
$$

given by $\operatorname{tr}_{P}^{S}(f)=\sum_{g_{i} \in X} g_{i} f g_{i}^{-1}$, where $X$ is a set of representatives for $S / P$. Each $\varphi: P \rightarrow S$ in $\mathcal{F}$ also induces a map

$$
\varphi^{*}: \operatorname{Hom}_{\mathbb{F}_{p}[S]}\left(M_{r}, C^{s}\left(S U(n) ; \mathbb{F}_{p}\right)\right) \rightarrow \operatorname{Hom}_{\mathbb{F}_{p}[P]}\left({ }^{\varphi} M_{r},{ }^{\varphi} C^{s}\left(S U(n) ; \mathbb{F}_{p}\right)\right)
$$

where ${ }^{\varphi} N$ denotes $N$ with the action of $P$ through $\varphi$. Recall that $\varphi$ induces a map $\bar{\varphi}: S U(n) / P \rightarrow S U(n) / S$ given by $x P \mapsto A x A^{-1} S$, where $A \in S U(n)$ is such that $A \rho(p) A^{-1}=\rho(\varphi(p))$ for all $p \in P$. Given $f: S U(n) \rightarrow S U(n)$, let us denote by $f^{\#}$ the induced endomorphism of $C^{s}\left(S U(n) ; \mathbb{F}_{p}\right)$. And given $B$ in $S U(n)$, we denote by $L_{B}: S U(n) \rightarrow S U(n)$ the map given by left multiplication by $B$. The map $c_{A}: S U(n) \rightarrow S U(n)$ that sends $X$ to $A X A^{-1}$ defines a $P$-equivariant map

$$
c_{A}^{\#}:{ }^{\varphi} C^{s}\left(S U(n) ; \mathbb{F}_{p}\right) \rightarrow C^{s}\left(S U(n) ; \mathbb{F}_{p}\right) .
$$

To see this, note that the action of $p \in P$ on ${ }^{\varphi} C^{s}\left(S U(n) ; \mathbb{F}_{p}\right)$ is given by $L_{\rho(\varphi(p))}^{\#}$ and by $L_{\rho(p)}^{\#}$ on $C^{s}\left(S U(n) ; \mathbb{F}_{p}\right)$. And we have

$$
c_{A}^{\#} L_{\rho(\varphi(p))}^{\#}=\left(L_{\rho(\varphi(p))} \circ c_{A}\right)^{\#}=\left(c_{A} \circ L_{\rho(p)}\right)^{\#}=L_{\rho(p)}^{\#} c_{A}^{\#},
$$

where the second equality holds because

$$
L_{\rho(\varphi(p))} c_{A}(B)=\rho(\varphi(p)) A B A^{-1}=A \rho(p) A^{-1} A B A^{-1}=c_{A} L_{\rho(p)}(B) .
$$

Therefore $c_{A}^{\#}$ induces a map

$$
\operatorname{Hom}_{\mathbb{F}_{p}[P]}\left({ }^{\varphi} M_{r},{ }^{\varphi} C^{s}\left(S U(n) ; \mathbb{F}_{p}\right)\right) \rightarrow \operatorname{Hom}_{\mathbb{F}_{p}[P]}\left({ }^{\varphi} M_{r}, C^{s}\left(S U(n) ; \mathbb{F}_{p}\right)\right) .
$$

And we denote by $\widetilde{\varphi}^{*}$ the composition of $\varphi^{*}$ with this map, that is:

$$
\widetilde{\varphi}^{*}: \operatorname{Hom}_{\mathbb{F}_{p}[S]}\left(M_{r}, C^{s}\left(S U(n) ; \mathbb{F}_{p}\right)\right) \rightarrow \operatorname{Hom}_{\mathbb{F}_{p}[P]}\left(M_{r}, C^{s}\left(S U(n) ; \mathbb{F}_{p}\right)\right) .
$$

Since the maps $\operatorname{tr}_{P}^{S}$ and $\widetilde{\varphi}^{*}$ commute with the differentials, we obtain an endomorphism of the double complex $C^{*, *}(S)$

$$
\widetilde{\Omega}^{*}=\sum_{(P, \varphi) \in \Omega} n_{P, \varphi} \operatorname{tr}_{P}^{S} \widetilde{\varphi}^{*}
$$

Therefore, we have an induced endomorphism of each term $E_{k}(S)$ in the spectral sequence, which we denote by $\Omega_{k}^{*}$. By construction, the image of $\Omega_{k}^{*}$ consists of the stable elements, hence

$$
\operatorname{Im}\left(\Omega_{k}^{*}\right) \cong \lim _{\mathcal{O}_{\left(\mathcal{F}^{c}\right)}} E_{k}(P)
$$

for all $k$.

Left multiplication of $S$ on $S U(n)$ via $\rho$ induces the action of $S$ on the cohomology groups of $S U(n)$ associated to the fibration $S U(n) \rightarrow S U(n) / S \rightarrow B S$. But left multiplication via $\rho$ factors through the left multiplication action of $S U(n)$ on itself and since $S U(n)$ is connected, the map given by left multiplication by an element of $S U(n)$ is homotopic to the identity map. Therefore the action of $S$ on $H^{*}\left(S U(n) ; \mathbb{F}_{p}\right)$ is trivial and so we have

$$
E_{2}^{r, s}(S) \cong H^{r}\left(B S ; \mathbb{F}_{p}\right) \otimes H^{s}\left(S U(n) ; \mathbb{F}_{p}\right) .
$$


Note that by construction, $\Omega_{2}^{*}$ coincides with the morphism $[\Omega]$ from Proposition 5.5 of 6 on $H^{*}\left(B S ; \mathbb{F}_{p}\right)$ and it is the identity on the $H^{s}\left(S U(n) ; \mathbb{F}_{p}\right)$-factor since $c_{A}^{*}$ is the identity. In particular, the choice of $A$ does not affect $\Omega_{k}^{*}$ for $k \geq 2$.

Consider now the cohomological Serre spectral sequence $E_{*}$ associated to the fibration $S U(n)_{p}^{\wedge} \rightarrow F \rightarrow|\mathcal{L}|_{p}^{\wedge}$. The action of the fundamental group of $|\mathcal{L}|_{p}^{\wedge}$ on $H^{*}\left(S U(n)_{p}^{\wedge} ; \mathbb{F}_{p}\right) \cong H^{*}\left(S U(n) ; \mathbb{F}_{p}\right)$ factors through the action of $S$, and so it is also trivial. Therefore the $E_{2}$-term is given by

$$
E_{2}^{r, s} \cong H^{r}\left(|\mathcal{L}|_{p}^{\wedge} ; \mathbb{F}_{p}\right) \otimes H^{s}\left(S U(n) ; \mathbb{F}_{p}\right) .
$$

The map $\Theta: B S \rightarrow|\mathcal{L}|_{p}^{\wedge}$ induces a map of fibre sequences

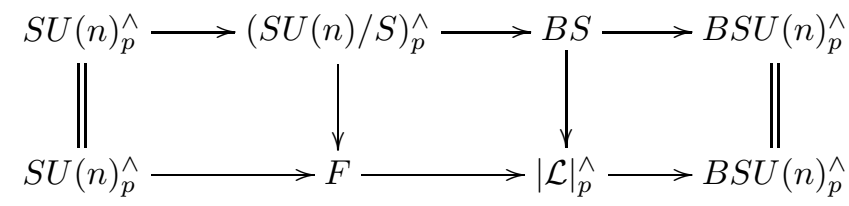

which in turn induces a morphism of the associated Serre spectral sequences. Considering the maps involved in this diagram, the corresponding morphism of spectral sequences $E_{2} \rightarrow E_{2}(S)$ is the morphism induced by the map $\Theta: B S \rightarrow|\mathcal{L}|_{p}^{\wedge}$ on the first factor of the tensor product and the identity on the second factor. Since the cohomology of a $p$-local finite group is computed by stable elements, the image of $E_{2} \rightarrow E_{2}(S)$ is precisely $\operatorname{Im}([\Omega]) \otimes H^{s}\left(S U(n) ; \mathbb{F}_{p}\right)$, which coincides with $\operatorname{Im}\left(\Omega_{2}^{*}\right)$. And therefore $E_{k} \cong \operatorname{Im}\left(\Omega_{k}^{*}\right)$ for all $k$.

Since $S U(n)$ is finite-dimensional, the spectral sequences $E_{*}$ and $E_{*}(S)$ collapse at a finite stage and therefore

$$
E_{\infty} \cong \operatorname{Im}\left(\Omega_{\infty}^{*}\right) \cong \lim _{\mathcal{O}\left(\mathcal{F}^{c}\right)} E_{\infty}(P)
$$

Now $E_{*}$ converges to $H^{*}\left(F ; \mathbb{F}_{p}\right)$ and $E_{*}(P)$ converges to $H^{*}\left(S U(n) / P ; \mathbb{F}_{p}\right)$. The endomorphism $\Omega_{\infty}^{*}$ of $E_{\infty}(P)$ coincides with the one induced by $\bar{\Omega}^{*}$ from Lemma 6.3 because the maps $c_{A}$ induce the maps $S U(n) / P \rightarrow S U(n) / S$ that take $x P$ to $A x A^{-1} S$. Therefore we have an isomorphism of cohomology groups

$$
H^{*}\left(F ; \mathbb{F}_{p}\right) \cong \lim _{\mathcal{O}\left(\mathcal{F}^{c}\right)} H^{*}\left(S U(n) / P ; \mathbb{F}_{p}\right)
$$

The action of $S$ on $S U(n)$ via $\rho$ is free and trivial on cohomology, hence $S U(n) / S$ is an orientable manifold. The same holds for any $P \leq S$. It remains to show that the fundamental class $\omega_{S} \in H^{N}\left(S U(n) / S ; \mathbb{F}_{p}\right)$ is stable, where $N$ is the dimension of $S U(n)$. For each $P<S$, the quotient $S U(n) / P \rightarrow S U(n) / S$ is a covering map of $p$-power index, hence the induced map in the $N$ th $\mathbb{F}_{p}$-cohomology group is zero. Therefore

$$
H^{N}\left(F ; \mathbb{F}_{p}\right) \cong \lim _{\mathcal{O}\left(\mathcal{F}^{c}\right)} H^{N}\left(S U(n) / P ; \mathbb{F}_{p}\right) \cong H^{N}\left(S U(n) / S ; \mathbb{F}_{p}\right)^{\operatorname{Aut}_{\mathcal{F}}(S)}
$$

But the action of an element $\varphi \in \operatorname{Aut}_{\mathcal{F}}(S)$ on $S U(n) / S$ factors through the action of $S U(n)$ on $S U(n) / S$ by conjugation, and so it is trivial on cohomology. Therefore

$$
H^{N}\left(F ; \mathbb{F}_{p}\right) \cong H^{N}\left(S U(n) / S ; \mathbb{F}_{p}\right)
$$

and in particular, the fundamental class of $S U(n) / S$ is stable.

Corollary 6.6. $H^{*}\left(F ; \mathbb{F}_{p}\right)$ is a Poincaré duality algebra. 
Proof. Let $N$ be dimension of $S U(n)$. By Proposition 6.5 and Remark 6.4 we know that $H^{*}\left(F ; \mathbb{F}_{p}\right) \cong H^{*}\left(S U(n) / S ; \mathbb{F}_{p}\right)^{\mathcal{F}}$. In particular, the cohomology groups $H^{i}\left(F ; \mathbb{F}_{p}\right)$ vanish for $i>N$. Proposition 6.5 also showed that the fundamental class $\omega_{S} \in H^{N}\left(S U(n) / S ; \mathbb{F}_{p}\right)$ is a stable element and $H^{N}\left(F ; \mathbb{F}_{p}\right) \cong H^{N}\left(S U(n) / S ; \mathbb{F}_{p}\right)$. Since $H^{*}\left(S U(n) / S ; \mathbb{F}_{p}\right)$ is a Poincaré duality algebra, the following diagram defines a pairing for $H^{*}\left(S U(n) / S ; \mathbb{F}_{p}\right)^{\mathcal{F}}$

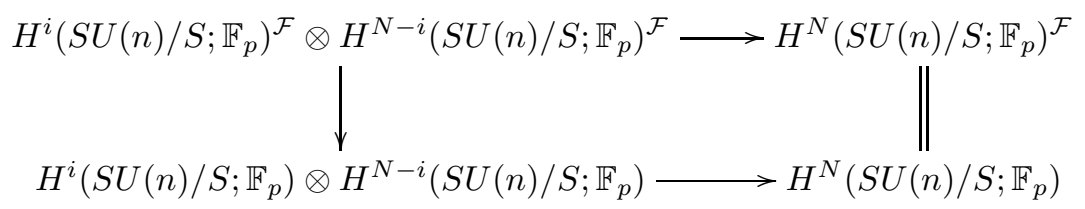

It remains to show that this pairing is non-singular. It is enough to show that for any $a$ in $H^{i}\left(S U(n) / S ; \mathbb{F}_{p}\right)^{\mathcal{F}}$, there exists $b \in H^{N-i}\left(S U(n) / S ; \mathbb{F}_{p}\right)^{\mathcal{F}}$ such that $a \smile b=\omega_{S}$. Since $H^{i}\left(S U(n) / S ; \mathbb{F}_{p}\right)$ is a Poincaré duality algebra, there exists $b^{\prime} \in H^{N-i}\left(S U(n) / S ; \mathbb{F}_{p}\right)$ such that $a \smile b^{\prime}=\omega_{S}$. Consider the element $b=\bar{\Omega}^{*}\left(b^{\prime}\right)$ in $H^{N-i}\left(S U(n) / S ; \mathbb{F}_{p}\right)^{\mathcal{F}}$. By Remark 6.4, the morphism $\bar{\Omega}^{*}$ is $H^{*}\left(S U(n) / S ; \mathbb{F}_{p}\right)^{\mathcal{F}_{-}}$ linear and so

$$
a \smile \bar{\Omega}^{*}\left(b^{\prime}\right)=\bar{\Omega}^{*}\left(a \smile b^{\prime}\right)=\bar{\Omega}^{*}\left(\omega_{S}\right)=\omega_{S},
$$

where the last equality holds because $\omega_{S}$ is a stable element.

Recall that a map $R \rightarrow k$ of differential graded algebras is Gorenstein of shift $a$ if there is a quasi-isomorphism $\operatorname{Hom}_{R}(k, R) \sim \Sigma^{a} k$ of differential graded algebras over $k$ and the natural map

$$
\operatorname{Hom}_{R}(k, R) \otimes_{\operatorname{End}_{R}(k)} \operatorname{Hom}_{R}(k, k) \rightarrow \operatorname{Hom}_{R}\left(k, \operatorname{Hom}_{R}(k, R) \otimes_{\operatorname{End}_{R}(k)} k\right)
$$

is a quasi-isomorphism of differential graded algebras over $\operatorname{End}_{R}(k)$. This is a particular case of Definition 8.1 from Dwyer-Greenlees-Iyengar [12].

As a consequence of Corollary 6.6, we obtain that $C^{*}\left(|\mathcal{L}|_{p}^{\wedge} ; \mathbb{F}_{p}\right) \rightarrow \mathbb{F}_{p}$ is Gorenstein. The proof follows the argument in [12, Example 10.3], and we refer to this article for the relevant notions which appear in this proof and the following results.

Theorem 6.7. Let $(S, \mathcal{F}, \mathcal{L})$ be a p-local finite group. Then the augmentation $C^{*}\left(|\mathcal{L}|_{p}^{\wedge} ; \mathbb{F}_{p}\right) \rightarrow \mathbb{F}_{p}$ is Gorenstein.

Proof. By Theorem 6.2, there is a homotopy monomorphism $|\mathcal{L}|_{p}^{\wedge} \rightarrow B S U(m)_{p}^{\wedge}$ for some $m \geq 0$. Let $F$ be the homotopy fibre of this map. Note that $C^{*}\left(X_{p}^{\wedge} ; \mathbb{F}_{p}\right)$ is quasi-isomorphic to $C^{*}\left(X ; \mathbb{F}_{p}\right)$ if $X$ is $p$-good. In particular, this holds for $|\mathcal{L}|_{p}^{\wedge}$, $B S U(m)$ and $S U(m)$.

By Proposition 6.5, all the homology groups $H_{k}\left(F ; \mathbb{F}_{p}\right)$ are finite-dimensional. And since $B S U(m)_{p}^{\wedge}$ and $S U(m)_{p}^{\wedge}$ are simply connected, the fundamental group of $F$ is isomorphic to the fundamental group of $|\mathcal{L}|_{p}^{\wedge}$. This is a finite $p$-group by Proposition 1.12 in 6 . Therefore $\left(F, \mathbb{F}_{p}\right)$ is of Eilenberg-Moore type.

Since $H^{*}\left(F ; \mathbb{F}_{p}\right)$ is finite-dimensional, Remark $5.5(2)$ in [12] tells us that the augmentation $C^{*}\left(F ; \mathbb{F}_{p}\right) \rightarrow \mathbb{F}_{p}$ is cosmall. By Remark 4.15 in [12], it is also proxysmall. Now Proposition 8.12 from [12] and Corollary 6.6 above imply that this augmentation is Gorenstein.

Theorem 7.14 in 20] shows that there is a quasi-isomorphism

$$
C^{*}\left(F ; \mathbb{F}_{p}\right) \sim C^{*}\left(|\mathcal{L}|_{p}^{\wedge} ; \mathbb{F}_{p}\right) \otimes_{C^{*}\left(B S U(m)_{p}^{\wedge} ; \mathbb{F}_{p}\right)} \mathbb{F}_{p} .
$$


By Section 10.2 in [12, the augmentation $C^{*}\left(B S U(m)_{p}^{\wedge} ; \mathbb{F}_{p}\right) \rightarrow \mathbb{F}_{p}$ is small and Gorenstein. Since the augmentation $C^{*}\left(B S U(m)_{p}^{\wedge} ; \mathbb{F}_{p}\right) \rightarrow \mathbb{F}_{p}$ is small and the morphism $C^{*}\left(F ; \mathbb{F}_{p}\right) \rightarrow \mathbb{F}_{p}$ is proxy-small, the augmentation $C^{*}\left(|\mathcal{L}|_{p}^{\wedge} ; \mathbb{F}_{p}\right) \rightarrow \mathbb{F}_{p}$ is proxy-small by Proposition 4.18 in [12].

The fibration $F \rightarrow|\mathcal{L}|_{p}^{\wedge} \rightarrow B S U(m)_{p}^{\wedge}$ is admissible in the sense of DwyerWilkerson [13], hence $C^{*}\left(|\mathcal{L}|_{p}^{\wedge} ; \mathbb{F}_{p}\right)$ is small over $C^{*}\left(B S U(m)_{p}^{\wedge} ; \mathbb{F}_{p}\right)$ by Lemma 2.10 in [13. The augmentation maps $C^{*}\left(B S U(m)_{p}^{\wedge} ; \mathbb{F}_{p}\right) \rightarrow \mathbb{F}_{p}$ and $C^{*}\left(F ; \mathbb{F}_{p}\right) \rightarrow \mathbb{F}_{p}$ are both Gorenstein, so we can use Proposition 8.10 from [12] to conclude that $C^{*}\left(|\mathcal{L}|_{p}^{\wedge} ; \mathbb{F}_{p}\right) \rightarrow \mathbb{F}_{p}$ is Gorenstein.

Corollary 6.8. Let $(S, \mathcal{F}, \mathcal{L})$ be a p-local finite group and let $\Omega\left(|\mathcal{L}|_{p}^{\wedge}\right)$ denote the based loopspace of $|\mathcal{L}|_{p}^{\wedge}$. Then the augmentation $C_{*}\left(\Omega\left(|\mathcal{L}|_{p}^{\wedge}\right) ; \mathbb{F}_{p}\right) \rightarrow \mathbb{F}_{p}$ is Gorenstein.

Proof. Since $\left(|\mathcal{L}|_{p}^{\wedge}, \mathbb{F}_{p}\right)$ is of Eilenberg-Moore type, it is dc-complete (see Section 4.22 in [12). We saw in the proof of the previous theorem that the augmentation $C^{*}\left(|\mathcal{L}|_{p}^{\wedge} ; \mathbb{F}_{p}\right) \rightarrow \mathbb{F}_{p}$ is proxy-small and Gorenstein. By Proposition 8.5 in [12, we conclude that $C_{*}\left(\Omega\left(|\mathcal{L}|_{p}^{\wedge}\right) ; \mathbb{F}_{p}\right) \rightarrow \mathbb{F}_{p}$ is Gorenstein.

Moreover, as in [12, Example 10.3], we get other interesting consequences, such as the existence of a local cohomology spectral sequence.

Corollary 6.9. Let $(S, \mathcal{F}, \mathcal{L})$ be a $p$-local finite group. There is a spectral sequence

$$
E_{i, j}^{2}=H_{I}^{-i}\left(H^{*}\left(|\mathcal{L}|_{p}^{\wedge} ; \mathbb{F}_{p}\right)\right)_{j} \Rightarrow H_{i+j}\left(|\mathcal{L}|_{p}^{\wedge} ; \mathbb{F}_{p}\right)
$$

where $I$ is the ideal of elements of positive dimension.

Proof. Since $C^{*}\left(|\mathcal{L}|_{p}^{\wedge} ; \mathbb{F}_{p}\right)$ is coconnective and connected, it follows by Remark 3.17 in [12] that $C_{*}\left(|\mathcal{L}|_{p}^{\wedge} ; \mathbb{F}_{p}\right)$ is $\mathbb{F}_{p}$-cellular over $C^{*}\left(|\mathcal{L}|_{p}^{\wedge} ; \mathbb{F}_{p}\right)$. Since the $\mathbb{F}_{p}$-cohomology of $|\mathcal{L}|_{p}^{\wedge}$ is Noetherian, it follows from Proposition 9.3 in [12] that there is a spectral sequence

$$
E_{i, j}^{2}=H_{I}^{-i}\left(H^{*}\left(|\mathcal{L}|_{p}^{\wedge} ; \mathbb{F}_{p}\right)\right)_{j} \Rightarrow H_{i+j-a}\left(|\mathcal{L}|_{p}^{\wedge} ; \mathbb{F}_{p}\right)
$$

where $I$ is the ideal of elements of positive dimension and $C^{*}\left(|\mathcal{L}|_{p}^{\wedge} ; \mathbb{F}_{p}\right) \rightarrow \mathbb{F}_{p}$ is Gorenstein of shift $a$. By Corollary 6.6, $F$ is a Poincaré duality algebra of the same dimension of $S U(m)$, so the shift of $C^{*}\left(B S U(m)_{p}^{\wedge} ; \mathbb{F}_{p}\right) \rightarrow \mathbb{F}_{p}$ and $C^{*}\left(F ; \mathbb{F}_{p}\right) \rightarrow \mathbb{F}_{p}$ coincide. By Propositions 8.6 and 8.10 in [12], the shift of $C^{*}\left(|\mathcal{L}|_{p}^{\wedge} ; \mathbb{F}_{p}\right) \rightarrow \mathbb{F}_{p}$ is zero.

Recall that a graded commutative Noetherian local ring $R$ with maximal ideal $\mathfrak{m}$ and residue field $k$ is Cohen-Macaulay if its local cohomology is concentrated in one degree. In this case, $R$ is Gorenstein if the local cohomology in this degree is isomorphic to $\operatorname{Hom}_{k}(R, k)$ (see Greenlees-Lyubeznik [16] for instance).

The local cohomology spectral sequence has structural implications on the cohomology of $|\mathcal{L}|_{p}^{\wedge}$. For example, if $H^{*}\left(|\mathcal{L}|_{p}^{\wedge} ; \mathbb{F}_{p}\right)$ is Cohen-Macaulay, then the spectral sequence collapses to give an isomorphism

$$
H_{I}^{r}\left(H^{*}\left(|\mathcal{L}|_{p}^{\wedge} ; \mathbb{F}_{p}\right)\right) \cong H_{*}\left(|\mathcal{L}|_{p}^{\wedge} ; \mathbb{F}_{p}\right) \cong \operatorname{Hom}_{\mathbb{F}_{p}}\left(H^{*}\left(|\mathcal{L}|_{p}^{\wedge} ; \mathbb{F}_{p}\right), \mathbb{F}_{p}\right)
$$

and so it is Gorenstein (see Greenlees [15] and Greenlees-Lyubeznik [16]).

Example 6.10. Some important examples of exotic 2-local finite groups were constructed in Levi-Oliver [19] (see also Benson [4]), motivated by the work of Solomon 28 of classifying all finite simple groups whose 2-Sylow subgroups are 
isomorphic to those of the Conway group $\mathrm{Co}_{3}$. They construct a 2-local finite group $\left(S, \mathcal{F}_{\text {Sol }}(q), \mathcal{L}_{\text {Sol }}^{c}(q)\right)$ over a 2-Sylow subgroup $S$ of $\operatorname{Spin}_{7}(q)$ for any odd prime power $q$. The $\mathbb{F}_{2}$-cohomology of these 2-local finite groups was computed by Grbić [14] to be

$$
H^{*}\left(\left|\mathcal{L}_{\text {Sol }}^{c}(q)\right|_{2}^{\wedge} ; \mathbb{F}_{2}\right) \cong \mathbb{F}_{2}\left[u_{8}, u_{12}, u_{14}, u_{15}, y_{7}, y_{11}, y_{13}\right] / I
$$

where $I$ is the ideal generated by the polynomials

$$
\begin{gathered}
y_{11}^{2}+u_{8} y_{7}^{2}+u_{15} y_{7}, \\
y_{13}^{2}+u_{12} y_{7}^{2}+u_{15} y_{11}, \\
y_{7}^{4}+u_{14} y_{7}^{2}+u_{15} y_{13} .
\end{gathered}
$$

In fact, Proposition 1 in [14] shows that $H^{*}\left(\left|\mathcal{L}_{\text {Sol }}^{c}(q)\right|_{2}^{\wedge} ; \mathbb{F}_{2}\right)$ is a finitely generated free $\mathbb{F}_{2}\left[u_{8}, u_{12}, u_{14}, u_{15}\right]$-module. Therefore the cohomology ring is Cohen-Macaulay (see Definition 5.4.9 and Theorem 5.4.10 in Benson [2]). Hence our arguments above imply that it must be Gorenstein.

In this particular case we can actually deduce that it is Gorenstein from the computation. The quotient of $H^{*}\left(\left|\mathcal{L}_{\text {Sol }}^{c}(q)\right|_{2}^{\wedge} ; \mathbb{F}_{2}\right)$ by the ideal generated by the polynomial subring $\mathbb{F}_{2}\left[u_{8}, u_{12}, u_{14}, u_{15}\right]$ is the graded ring

$$
\mathbb{F}_{2}\left[y_{7}, y_{11}, y_{13}\right] /\left(y_{11}^{2}, y_{13}^{2}, y_{7}^{4}\right)
$$

which is a Poincaré duality algebra. By Proposition I.1.4 and the Remark on the same page of Meyer-Smith [21, we can conclude that $H^{*}\left(\left|\mathcal{L}_{\text {Sol }}^{c}(q)\right|_{2}^{\wedge} ; \mathbb{F}_{2}\right)$ is Gorenstein.

\section{REFERENCES}

1. J. F. Adams, Maps between classifying spaces. II, Invent. Math. 49 (1978), no. 1, 1-65. MR 511095

2. D. J. Benson, Representations and cohomology. II, Cambridge Studies in Advanced Mathematics, vol. 31, Cambridge University Press, Cambridge, 1991, Cohomology of groups and modules. MR 1156302

3. D. J. Benson and Jon F. Carlson, Projective resolutions and Poincaré duality complexes, Trans. Amer. Math. Soc. 342 (1994), no. 2, 447-488. MR 1142778

4. David J. Benson, Cohomology of sporadic groups, finite loop spaces, and the Dickson invariants, Geometry and cohomology in group theory (Durham, 1994), London Math. Soc. Lecture Note Ser., vol. 252, Cambridge Univ. Press, Cambridge, 1998, pp. 10-23. MR 1709949

5. Carles Broto, Natàlia Castellana, Jesper Grodal, Ran Levi, and Bob Oliver, Subgroup families controlling p-local finite groups, Proc. London Math. Soc. (3) 91 (2005), no. 2, 325-354. MR 2167090

6. Carles Broto, Ran Levi, and Bob Oliver, The homotopy theory of fusion systems, J. Amer. Math. Soc. 16 (2003), no. 4, 779-856. MR 1992826

7. groups, Geom. Topol. 11 (2007), 315-427. MR 2302494

8. José Cantarero and Natàlia Castellana, Unitary embeddings of finite loop spaces, Forum Math. 29 (2017), no. 2, 287-311. MR 3619114

9. Natàlia Castellana and Assaf Libman, Wreath products and representations of p-local finite groups, Adv. Math. 221 (2009), no. 4, 1302-1344. MR 2518640

10. Andrew Chermak, Fusion systems and localities, Acta Math. 211 (2013), no. 1, 47-139. MR 3118305

11. W. Dwyer and A. Zabrodsky, Maps between classifying spaces, Algebraic topology, Barcelona, 1986, Lecture Notes in Math., vol. 1298, Springer, Berlin, 1987, pp. 106-119. MR 928826

12. W. G. Dwyer, J. P. C. Greenlees, and S. Iyengar, Duality in algebra and topology, Adv. Math. 200 (2006), no. 2, 357-402. MR 2200850

13. W. G. Dwyer and C. W. Wilkerson, The fundamental group of a p-compact group, Bull. Lond. Math. Soc. 41 (2009), no. 3, 385-395. MR 2506823 
14. Jelena Grbić, The cohomology of exotic 2-local finite groups, Manuscripta Math. 120 (2006), no. 3, 307-318. MR 2243565

15. J. P. C. Greenlees, Local cohomology in equivariant topology, Local cohomology and its applications (Guanajuato, 1999), Lecture Notes in Pure and Appl. Math., vol. 226, Dekker, New York, 2002, pp. 1-38. MR 1888194

16. J. P. C. Greenlees and G. Lyubeznik, Rings with a local cohomology theorem and applications to cohomology rings of groups, J. Pure Appl. Algebra 149 (2000), no. 3, 267-285. MR 1762768

17. Stefan Jackowski and Bob Oliver, Vector bundles over classifying spaces of compact Lie groups, Acta Math. 176 (1996), no. 1, 109-143. MR 1395671

18. Michael A. Jackson, A quotient of the set $[B G, B \mathrm{U}(n)]$ for a finite group $G$ of small rank, J. Pure Appl. Algebra 188 (2004), no. 1-3, 161-174. MR 2030812

19. Ran Levi and Bob Oliver, Construction of 2-local finite groups of a type studied by Solomon and Benson, Geom. Topol. 6 (2002), 917-990. MR 1943386

20. John McCleary, A user's guide to spectral sequences, second ed., Cambridge Studies in Advanced Mathematics, vol. 58, Cambridge University Press, Cambridge, 2001. MR 1793722

21. Dagmar M. Meyer and Larry Smith, Poincaré duality algebras, Macaulay's dual systems, and Steenrod operations, Cambridge Tracts in Mathematics, vol. 167, Cambridge University Press, Cambridge, 2005. MR 2177162

22. Haynes Miller, The Sullivan conjecture on maps from classifying spaces, Ann. of Math. (2) 120 (1984), no. 1, 39-87. MR 750716

23. Stephen A. Mitchell, On p-adic topological $K$-theory, Algebraic $K$-theory and algebraic topology (Lake Louise, AB, 1991), NATO Adv. Sci. Inst. Ser. C Math. Phys. Sci., vol. 407, Kluwer Acad. Publ., Dordrecht, 1993, pp. 197-204. MR 1367298

24. Bob Oliver, Fixed point sets and tangent bundles of actions on disks and Euclidean spaces, Topology 35 (1996), no. 3, 583-615. MR 1396768

25. - Existence and uniqueness of linking systems: Chermak's proof via obstruction theory, Acta Math. 211 (2013), no. 1, 141-175. MR 3118306

26. Lluis Puig, Frobenius categories, J. Algebra 303 (2006), no. 1, 309-357. MR 2253665

27. Kári Ragnarsson, Classifying spectra of saturated fusion systems, Algebr. Geom. Topol. 6 (2006), 195-252. MR 2199459

28. Ronald Solomon, Finite groups with Sylow 2-subgroups of type 3, J. Algebra 28 (1974), 182198. MR 0344338

29. Zdzisław Wojtkowiak, On maps from ho $\lim _{\longrightarrow} F$ to Z A Algebraic topology, Barcelona, 1986, Lecture Notes in Math., vol. 1298, Springer, Berlin, 1987, pp. 227-236. MR 928836

Consejo Nacional de Ciencia y Tecnología,

Centro de Investigación en Matemáticas, A.C. Unidad Mérida,

Parque Científico y TeCnológico de Yucatán,

Carretera Sierra Papacal-Chuburná Km 5.5,

MÉRIDA, YUC 97302,

MeXiCO.

E-mail address: cantarero@cimat.mx

Departament de Matemàtiques,

Universitat Autònoma de Barcelona and BGSMath,

EDIFICI CC,

E-08193 Bellaterra,

SPAIN.

E-mail address: natalia@mat.uab.cat

IES ClaRA CAMPOAMOR,

Av. DE Alcorcón, 1,

E-28936 Móstoles,

SPAIN. 